\title{
DUNE prospects in the search for sterile neutrinos
}

\author{
Igor Krasnov $\odot^{*}$ \\ Institute for Nuclear Research of the Russian Academy of Sciences, \\ 60th October Anniversary Prospect 7a, 117312 Moscow, Russia
}

(Received 19 February 2019; revised manuscript received 18 July 2019; published 18 October 2019)

\begin{abstract}
Experiments measuring the parameters of active neutrino oscillations can also search for the sterile neutrinos in part of the sterile neutrino parameter space. We analyze the prospects for the sterile neutrino search in the upcoming experiment DUNE for the sterile neutrinos with masses at GeV scale. As the experiment relies on the still-undecided design of the Near Detector, we provide the expected number of sterile neutrino decays in the Near Detector volume. Our most optimistic predictions show that the corresponding limit on mixing can be approximately of the same order as the previous estimates made for the LBNE project. We present our results as separate plots for the sterile neutrino mixing with electron, muon, and tau neutrinos. Generally, DUNE has good prospects for probing a large region of a previously unavailable part of the parameter space before new projects (like the SHiP one) join the search.
\end{abstract}

DOI: 10.1103/PhysRevD.100.075023

\section{INTRODUCTION}

Physics beyond the Standard Model (SM) of particle physics is one of the most rapidly developing fields in theoretical physics. It stems from the discrepancies between SM predictions and some of the experimental data obtained in recent decades. For example, neutrino oscillation phenomena show that the SM is not complete. One way to address this problem is to introduce additional leptons, sterile with respect to the SM gauge interactions $S U(3)_{c} \times$ $S U(2)_{W} \times U(1)_{Y}$ [1]. They are usually called sterile neutrinos and are introduced in the following way:

$\mathcal{L}=i \bar{N}_{I} \gamma^{\mu} \partial_{\mu} N_{I}-\left(\frac{1}{2} M_{I} \bar{N}_{I}^{c} N_{I}+Y_{\alpha I} \bar{L}_{\alpha} \tilde{H} N_{I}+\right.$ H.c. $) ;$

here $N_{I}$ are sterile neutrinos, $M_{I}$ are their Majorana masses, and $Y_{\alpha I}$ stand for their Yukawa couplings with lepton doublets $L_{\alpha}, \alpha=e, \mu, \tau$ and SM Higgs doublet $\left(\tilde{H}_{a}=\epsilon_{a b} H_{b}^{*}\right)$. One needs at least two sterile neutrinos to explain active neutrino oscillations, and at least three in the case where all active neutrinos have nonzero masses. It was shown that heavy sterile neutrinos may also provide an explanation for leptogenesis (see, for example, Ref. [2]) or serve as a dark matter candidate [3].

\footnotetext{
*iv.krasnov@physics.msu.ru
}

Published by the American Physical Society under the terms of the Creative Commons Attribution 4.0 International license. Further distribution of this work must maintain attribution to the author(s) and the published article's title, journal citation, and DOI. Funded by SCOAP ${ }^{3}$.
The strategy of the search for such particles depends heavily on their masses. If sterile neutrinos have masses at $\mathrm{GeV}$ scale, they can appear in heavy hadron decays. Such sterile neutrinos can be searched for in various collider experiments. Experiments measuring the parameters of neutrino oscillations are capable of detecting sterile neutrino decay events as well. Beam energies, as well as specifics of measurement processes, geometry, and the relative position of the detector, determine the region of sterile neutrino parameter space that can be tested in a given experiment. Experiments such as CHARM [4], NuTeV [5], PS191 [6], DELPHI [7], OKA [8], LHCb [9,10], Belle [10], and E949 [11] provide limits on active-sterile neutrino mixing. Many developing projects and upcoming experiments, such as NA62 [12,13], SHiP [14], MATHUSLA [15], T2K [16], and DUNE [17-20] declare the search for heavy neutral leptons to be one of their goals.

The search for the sterile neutrinos in beam-dump experiments was considered, for example, in Refs. [21,22]. The process behind the search can be described as follows: the proton beam strikes the target and produces a great number of heavy secondary mesons. Because of active-sterile neutrino mixing, a part of these mesons would produce sterile neutrinos in their decays. A part of these sterile neutrinos flies toward the detector and decays inside its volume. Such decays can be observed.

The LBNE project provided its estimate of active-sterile neutrino mixing by rescaling the results of existing experimental data using the new experiment specifics in their design report [23]. The DUNE project inherited this estimate as their own predicted sensitivity to active-sterile neutrino mixing without updating the specifics of the experiment, such as the Near Detector length. To date, 
there has been no update made on the proposed DUNE Near Detector design [17-20].

The aim of this paper is to calculate the sensitivity of DUNE to the active-sterile neutrino mixing for sterile neutrinos of masses at $\mathrm{GeV}$ scale. As it relies on the yet to be decided design of the Near Detector, it is impossible to provide a proper estimation of the number of background events. In this paper, we present the isocontours for the number of expected heavy neutrino decays inside the detector volume, in the plane $M_{N}-|U|^{2}$, thus avoiding the issue of dealing with the experimental detection efficiencies and reconstruction effects. We also propose some ideas as to how it might be possible to enhance the signal to background ratio. This paper could be useful for the consideration of the DUNE Near Detector design or its possible additional upgrades.

The paper is organized as follows. In Sec. II, we present the overall layout of DUNE Near Detector facilities and the relevant proton beam properties. After that, in Sec. III we list the experimental features of the search, such as different meson production rates and their momentum distribution. We present in more detail the analysis of sterile neutrino detection specifics and our way to account for it in Sec. IV. We present our estimates in Sec. V, and some possible issues in Sec. VI. We conclude in Sec. VII. We also present the various relevant experimental data in Appendix A and the sterile neutrino-related formulas in Appendix B.

\section{DUNE}

The main goal of DUNE is to measure active neutrino parameters with high precision [17-20]. This will be achieved by creating very intensive high-energy neutrino flux. A high-energy proton beam (up to $120 \mathrm{GeV}$ ) strikes a target, producing a high number of secondary particles (mainly pions and kaons) which can produce sterile neutrinos during their decay. To provide enough space for the secondary particles to decay, a $221 \mathrm{~m}$ long and $4 \mathrm{~m}$ wide decay pipe is planned to be installed behind the target area. At the end of the pipe, the absorber is placed to reduce the background from muons. Additionally, natural rock fills the area between the decay pipe and the detector. The resulting neutrino beam is directed toward the Near Detector at $574 \mathrm{~m}$ from the target and the Far Detector at $1300 \mathrm{~km}$, which allows for better prospects for active neutrino parameter measurement.

Important properties of a reference proton beam are listed in Table I. Geometrical sizes are listed in Table II.

As the Near Detector is located considerably far from the target, one can notice that the heavy sterile neutrino would reach the detector later than the active neutrinos produced at the same time. But that shift in arrival time is generally less than the active neutrino travel time from the target to the detector, $t_{\nu} \approx \frac{574 \mathrm{~m}}{3 \times 10^{8} \mathrm{~m} / \mathrm{s}}=1.91 \mu \mathrm{s}$. And the latter time is considerably less than the spill duration of $\tau=10 \mu \mathrm{s}$. We can use a timing cut only if the sterile neutrino arrives at the
TABLE I. Proton beam properties [19].

\begin{tabular}{lc}
\hline Proton beam energy & $120 \mathrm{GeV}$ \\
Spill duration & $1.0 \times 10^{-5} \mathrm{~s}$ \\
Protons on target per year & $1.1 \times 10^{21}$ \\
Cycle time & $1.2 \mathrm{~s}$ \\
\hline \hline
\end{tabular}

TABLE II. Geometrical sizes [19].

\begin{tabular}{lc}
\hline \hline Distance from the target to the near detector $L$ & $574 \mathrm{~m}$ \\
Decay pipe length $l_{\text {decay pipe }}$ & $194 \mathrm{~m}$ \\
Decay pipe radius $r_{\text {decay pipe }}$ & $2 \mathrm{~m}$ \\
Near Detector reference size & $6.4 \times 3.5 \times 3.5 \mathrm{~m}$ \\
$\Delta l \times \Delta h \times \Delta h[20]$ & \\
\hline \hline
\end{tabular}

detector after the last of the active neutrinos, produced during the spill, has passed through it. There are two random elements in this. First, if we start the time count at the beginning of the spill, the moment of the possible sterile neutrino production $t_{0}$ is evenly distributed between 0 and $\tau$. The other random element is the value of the sterile neutrino momentum. We present the way we construct the sterile neutrino momentum distribution in Sec. IV. The timing cut criterion for the sterile neutrino with velocity $v_{N}$ can be expressed as follows: $t_{0}+\frac{L}{v_{N}}>\tau+\frac{L}{c}$. Therefore the application of the timing cut is more probable for the sterile neutrinos produced at the end of the spill, but it also can be applied to the sterile neutrinos that were produced early if their momentum is sufficiently small. The timing cut can be used for all sterile neutrinos with mass $M_{N}$ and momentum $p<\frac{L}{\sqrt{(L+c \tau)^{2}-L^{2}}} M_{N}$. The probability $P$ for the timing cut to apply to the produced sterile neutrino with mass $M_{N}$ and fixed momentum $p>\frac{L}{\sqrt{(L+c \tau)^{2}-L^{2}}} M_{N}$ reads $P=\frac{L}{c \tau}\left(\sqrt{1+\frac{M_{N}^{2}}{p^{2}}}-1\right)$. Taking into account the sterile neutrino momentum distribution (see Sec. IV), we obtain the result that less than $0.1 \%$ of the sterile neutrinos that fly in the direction of the detector satisfy the timing cut criterion. This means that, for the most part, the active neutrino spill would overlap with the sterile neutrino arrival, serving as the background for the sterile neutrino search. We note that the active neutrinos are generally produced in the decays of pions or kaons. These mesons have considerable mass, and this means that their travel time before their decay, compared to our estimate, contains an additional delay in the active neutrino arrival time. The same reasoning applies to the sterile neutrinos produced in the kaon decays, which results in the additional delay in the sterile neutrino arrival time for these sterile neutrinos, although that delay is shorter than the delay for the active neutrinos produced in pion decays. Therefore our estimate is conservative. Summing up, timing does not help one to 
get rid of the background from active neutrino interactions in the Near Detector. However, a special run with much shorter spill duration and lower beam energy can be considered a solution to this problem.

The Far Detector is simply too far to provide a sufficient number of sterile neutrino decays in the detector volume. We discuss geometrical restrictions in Sec. IV.

\section{EXPERIMENTAL FEATURES}

We assume that the primary $120 \mathrm{GeV}$ proton beam strikes a target and produces mesons that may decay into the sterile neutrinos and SM particles. Sterile neutrino momentum $p_{N}$ and energy $E_{N}$ spectra are very important for further analysis. They are closely related to the momentum $p_{H}$ and energy $E_{H}$ spectra of secondary mesons. Each momentum has longitudinal $p_{L}$ and transversal $p_{T}$ components. The longitudinal component is the one directed along the axis of the active neutrino beam, and the transversal component is orthogonal to the longitudinal one.

It is shown in Ref. [21] that the number $d N_{H}$ of heavy hadrons is proportional to the differential cross section of the relevant hadron direct production $d \sigma_{H}$ :

$$
\frac{d N_{H}}{d p_{H_{L}} d p_{H_{T}}^{2}} \propto \frac{d \sigma_{H}}{d p_{H_{L}} d p_{H_{T}}^{2}} .
$$

The distribution of the longitudinal momentum of secondary mesons can be fitted from experimental data, with fit parameters varying with beam energy. The usual approximation for the longitudinal momentum $p_{H_{L}}$ distribution of the differential cross section $d \sigma_{H}$ reads $[24,25]$

$$
\frac{d \sigma_{H}}{d x_{F}} \propto\left(1-x_{F}\right)^{c}, \quad x_{F}=\frac{p_{H_{L}}}{p_{H_{L}}^{\max }},
$$

with $c=3$ being the phenomenological factor-of- 2 estimate for the relevant energy $E=120 \mathrm{GeV}$ (see [21]).

The distribution of the transversal momentum of secondary mesons depends heavily on the details of hadronization [21]. They are usually approximated with fragmentation function $D(z)$. We take PYTHIA distributions, i.e., the Lund fragmentation function [26]:

$$
D(z)=\frac{(1-z)^{a}}{z^{1+r_{Q} b m_{Q}^{2}}} \exp \left(-\frac{b}{z}\left(M_{H}^{2}+p_{H_{T}}^{2}\right)\right),
$$

where $z$ represents a part of hadron momentum $p_{H}$ carried by heavy quark $p_{Q}$. The default parameter values (the ones that we take) are $a=0.68, b=0.98 \mathrm{GeV}^{-2}, r_{s}=0$, $r_{c}=1.32, r_{b}=0.855$ [26]. Heavy quark masses are $m_{c}=1.275 \mathrm{GeV}, m_{b}=4.18 \mathrm{GeV}$ [27]. The resulting transversal momentum distribution of secondary mesons reads

$$
\frac{d \sigma_{H}}{d p_{H_{T}}^{2}} \propto \int_{0}^{1} d z \frac{(1-z)^{a}}{z^{1+r_{Q} b m_{Q}^{2}}} \exp \left(-\frac{b}{z}\left(M_{H}^{2}+p_{H_{T}}^{2}\right)\right) .
$$

Different mesons have different chances to be produced in a specific experiment. This is determined by two factors: how many quarks of the corresponding type $\chi_{q}$ are generated by interactions of the primary beam with the target and the weight of a specific channel in quark hadronization $\operatorname{Br}(q \rightarrow H \ldots)$. Basically, after the number of simplifications Eq. (2) is transformed into the following form [21]:

$$
N_{H}=N_{P O T} \times M_{p p} \times \chi_{q} \times \operatorname{Br}(q \rightarrow H),
$$

where $N_{H}$ is the number of secondary hadrons and $N_{P O T}$ is the total number of "protons on target" (we identify it with the total number of proton interactions in the thin target). $M_{p p}$ is the multiplicity of reaction, i.e., the number of the hadrons produced in the interaction of primary protons with the target. It is already taken into account for all considered mesons except $\mathrm{K}$ mesons in the value of $\chi_{q}$. We take $M_{p p}=1$ for these mesons; for K-mesons, the value $M_{p p}>1$ depends on the primary beam energy: $M_{p p}(K)=$ 11 for $E=120 \mathrm{GeV}$ [21]. We take the following values of $\chi_{q}[28]:$

$\chi_{s} \equiv \frac{\sigma_{p p \rightarrow s}}{\sigma_{p p_{\text {total }}}}=\frac{1}{7}, \quad \chi_{c} \equiv \frac{\sigma_{p p \rightarrow c}}{\sigma_{p p_{\text {total }}}}=10^{-4}$,

$\chi_{b} \equiv \frac{\sigma_{p p \rightarrow b}}{\sigma_{p p_{\text {total }}}}=10^{-10}$.

For $s$-quark production fractions, we take [21]

$\operatorname{Br}\left(s \rightarrow K^{-}\right)=\operatorname{Br}\left(s \rightarrow K_{L}^{0}\right)=\operatorname{Br}\left(s \rightarrow K_{S}^{0}\right)=1 / 3$.

For $c$-quark production fractions, we take [14]

$\operatorname{Br}\left(c \rightarrow D^{+}\right)=0.207, \quad \operatorname{Br}\left(c \rightarrow D^{0}\right)=0.632$,

$\operatorname{Br}\left(c \rightarrow D_{s}^{+}\right)=0.088$.

For $b$-quark production fractions, we take [27]

$$
\begin{aligned}
\operatorname{Br}\left(b \rightarrow B^{+}\right) & =\operatorname{Br}\left(b \rightarrow B^{0}\right)=0.405, \\
\operatorname{Br}\left(b \rightarrow B_{s}^{0}\right) & =0.101 .
\end{aligned}
$$

The production fraction $\operatorname{Br}\left(b \rightarrow B_{c}^{+}\right)$has been measured only at LHC energies, where it reaches a few $\times 10^{-3}$ [29]. At lower energies, it is not known. We take

$$
\operatorname{Br}\left(b \rightarrow B_{c}^{+}\right)=10^{-3} .
$$

Sterile neutrino production fractions from various mesons as well as sterile neutrino decay modes are listed in Appendix B. 


\section{ALGORITHM}

We want the detector to detect the sterile neutrino decay signals and distinguish them from the background. The main question is: how small can the value of $\left|U^{2}\right|$ be to still allow it? The answer to the question depends heavily on the detector configuration, its efficiency, and the methods used to reduce the background. As none of the above can be clarified yet, we provide only the isocontours for the number of expected heavy neutrino decays $N_{\text {detector }}$ inside the detector volume, in the plane $M_{N}-|U|^{2}$ (we plan to provide the proper estimation of the number of background events in another paper after the Near Detector design is fixed). We simply scan the values of $M_{N}$ with a $20 \mathrm{MeV}$ step, starting from $M_{N}=140 \mathrm{MeV}$. As we increase the value of $M_{N}$, we eventually reach the value for which the predicted number of expected heavy neutrino decays becomes less than the specified value $N_{\text {detector }}$ for a specific isocontour. We abort our scan at this mass value.

First, we calculate the energy distribution function in each process according to Eqs. (B8) and (B9) in Appendix B 2. Note that this is an energy in the rest frame of the decaying meson $H$. We also calculate the sterile neutrino mean lifetime $\tau_{N}=\frac{1}{\sum \Gamma(N \rightarrow \ldots)}$ according to Eqs. (B1)-(B7) in Appendix B 1.

After that, we randomly choose one of the processes, using the corresponding weight $\chi_{q} \times \operatorname{Br}(q \rightarrow H) \times$ $\operatorname{Br}(H \rightarrow N \ldots)$ according to formulas (B8), (B9), (B15), and (B17)-(B19) from Appendix B 2, and values (7)-(11). The following decays are significant: $K_{L}^{0} \rightarrow \pi^{+} l^{-} N$, $K^{+} \rightarrow \pi^{0} l^{+} N, \quad D^{0} \rightarrow K^{+} l^{-} N, \quad D^{+} \rightarrow K^{0} l^{+} N, \quad D^{0} \rightarrow$ $\pi^{+} l^{-} N, \quad D^{+} \rightarrow \pi^{0} l^{+} N, \quad D_{s}^{+} \rightarrow \eta^{0} l^{+} N, \quad B^{+} \rightarrow D^{0} l^{+} N$, $B^{0} \rightarrow D^{+} l^{-} N, \quad D^{0} \rightarrow K^{+*} l^{-} N, \quad D^{+} \rightarrow K^{0 *} l^{+} N, \quad B^{0} \rightarrow$ $D^{+*} l^{-} N, B^{+} \rightarrow D^{0 *} l^{+} N, B_{s}^{0} \rightarrow D_{s}^{+} l^{-} N, B_{s}^{0} \rightarrow D_{s}^{*+} l^{-} N$. The indirect production of the mesons from the decays of heavier mesons can be neglected, as their number is several orders of magnitude smaller than the number of the mesons produced in proton collisions in the target, as can be seen in Eqs. (6) and (7). We randomly choose $E_{N}$ from the previously calculated distribution that corresponds to the chosen process. From the distributions described by Eqs. (2) and (3), we randomly choose $p_{H_{L}}, p_{H_{T}}, p_{H}^{2}=$ $p_{H_{L}}^{2}+p_{H_{T}}^{2}$.

There is no preference for the direction of sterile neutrino momentum $p=\left\{p_{x}, p_{y}, p_{z}\right\}$ in the rest frame of $H$, so this direction is also chosen randomly. Its absolute value is $p=\sqrt{E_{N}^{2}-M_{N}^{2}}$. Then we make a Lorentz boost to this momentum to calculate the resulting sterile neutrino momentum $p_{N}$ in the laboratory frame. We choose the target as the point of origin; the $z$ axis is directed toward the detector, and the $x, y$ axes are chosen so that $p_{H_{L}} \equiv p_{H_{z}}$, $p_{H_{T}} \equiv p_{H_{x}}, p_{H_{y}}=0$. For convenience, we plot the scheme of the process geometry in Fig. 1. The resulting longitudinal and transversal components of sterile neutrino momentum in the laboratory frame read



FIG. 1. Schematic illustration of the detector geometry.

$$
\begin{gathered}
p_{N_{x}}=-\frac{E_{N}}{M_{H}} p_{H_{T}}-p_{z} \sqrt{1+\frac{p_{H}^{2}}{M_{H}^{2}}} \frac{p_{H_{T}}}{p_{H}}+p_{x} \frac{p_{H_{L}}}{p_{H}}, \\
p_{N_{y}}=p_{y}, \\
p_{N_{z}}=\frac{E_{N}}{M_{H}} p_{H_{L}}+p_{z} \sqrt{1+\frac{p_{H}^{2}}{M_{H}^{2}}} \frac{p_{H_{L}}}{p_{H}}+p_{x} \frac{p_{H_{T}}}{p_{H}} \equiv p_{N_{L}} .
\end{gathered}
$$

Note that, according to Eq. (14), if the decaying meson velocity in the laboratory frame $v_{H_{\mathrm{lab}}}=\frac{p_{H}}{E_{H}}$ is smaller than the sterile neutrino longitudinal velocity in the meson rest frame $v_{N_{L_{H}}}=\frac{p_{z}}{E_{N}}, v_{H_{\mathrm{lab}}}<v_{N_{L_{H}}}$, then it is possible that $p_{N_{z}}<0$, i.e., the sterile neutrino flies in the direction opposite that of the detector. Obviously, such a sterile neutrino will not be detected.

After the proton beam strikes the target, the produced secondary particles travel some distance away from the target before decaying. The distance that the $H$ meson travel down the pipe before decaying at the moment $t_{H}$ is $z_{H}=\frac{p_{H_{L}}}{M_{H}} t_{H}(t=0$ corresponds to the moment when the proton beam strikes the target). Its shift from the axis at this moment is $x_{H}=\frac{p_{H_{T}}}{M_{H}} t_{H}$. For short-lived mesons $t_{H} \sim 0$, $z_{H} \sim 0, x_{H} \sim 0$. If the meson produces sterile neutrino, its initial coordinates are $x_{N}\left(t_{H}\right)=x_{H}, \quad y_{N}\left(t_{H}\right)=0$, $z_{N}\left(t_{H}\right)=z_{H}$. One of the criteria for the sterile neutrino to decay in the detector volume is for it to decay when $L<z_{N}\left(t_{H}+\Delta t\right)<L+\Delta l$. Here $L$ is the distance from the target to the detector, $\Delta l$ is the effective length of the detector, and $\Delta t=\frac{M_{N}}{p_{N_{L}}}\left(L-z_{H}\right)$ is the time it takes for the sterile neutrino to travel the distance $L-z_{H}$. As $\Delta l \ll L$, we simply can take $z_{N}\left(t_{H}+\Delta t\right)=L$. The other sterile neutrino coordinates $x_{N}, y_{N}$ can be expressed as

$$
\begin{gathered}
x_{N}=\frac{p_{H_{T}}}{M_{H}} t_{H}+\frac{p_{N_{x}}}{p_{N_{z}}}\left(L-\frac{p_{H_{L}}}{M_{H}} t_{H}\right), \\
y_{N}=\frac{p_{N_{y}}}{p_{N_{z}}}\left(L-\frac{p_{H_{L}}}{M_{H}} t_{H}\right) .
\end{gathered}
$$


For the on-axis detector, we take the number of sterile neutrinos flying in the direction of the detector $\mathcal{N}_{\text {forward }}$ to be the number of sterile neutrinos for which the following statement is true:

$$
\sqrt{x_{N}^{2}+y_{N}^{2}}<\frac{\Delta h}{2}
$$

Here $\Delta h$ is the transversal size (height and width) of the detector. Equation (17) means that the sterile neutrino will not fly toward the detector if it deviates too much from axis $z$. For short-lived mesons $t_{H} \sim 0, z_{H} \sim 0$, Eq. (17) turns into

$$
\frac{p_{N_{T}}}{p_{N_{L}}}<\frac{\Delta h}{2 L}
$$

where $p_{N_{T}}=\sqrt{p_{N_{x}}^{2}+p_{N_{y}}^{2}}$ is the sterile neutrino transversal momentum.

Another random variable is the moment of decay of the meson $H$. Probability for meson $H$ to decay before the moment $t_{H}$ in the meson rest frame is

$$
P\left(t_{H}\right)=1-\exp \left(-\frac{t_{H}}{\tau_{H}}\right),
$$

where $\tau_{H}$ is the meson mean lifetime. We choose $t_{H}$ according to this law (19).

For long-lived mesons (kaons), we have an additional consideration: if the kaon longitudinal travel distance $z_{H}$ exceeds the decay pipe length $l_{\text {decay pipe }}, z_{H}>l_{\text {decay pipe }}$, then the kaon reaches the absorber. In pretty much the same way, if the kaon transversal travel distance $x_{H}=\frac{p_{H_{T}}}{M_{H}} t_{H}$ exceeds the decay pipe radius $r_{\text {decay pipe }}, x_{H}>r_{\text {decay pipe }}$, then it collides into the decay pipe walls. When either case happens, the kaon usually rapidly loses energy. At the moment of its decay, it practically stops. From Eqs. (15)(18), it is obvious that sterile neutrinos from such kaons are not very relativistic and have a very small probability of reaching the detector hundreds of meters away. For that reason, we consider the contribution of such kaons negligible and remove them from our estimates at the moment they reach the absorber or the walls.

We repeat this process many times (we take $\mathcal{N}_{\text {total }}=10^{7}$ iterations) and take into account only those events that satisfy criterion (17) and obtain the number of sterile neutrinos $\mathcal{N}_{\text {forward }}$ that fly in the direction of the detector. The portion of sterile neutrinos $\zeta_{N}$ that flies toward the detector reads

$$
\zeta_{N}=\frac{4}{\pi} \frac{\mathcal{N}_{\text {forward }}}{\mathcal{N}_{\text {total }}}
$$

where coefficient $\frac{4}{\pi}$ represents the fact that the frontal surface of the detector is a square and not a circle, as implied in Eq. (17).
We also obtain the resulting distribution $f_{1}\left(p_{N_{L}}\right)$ of the longitudinal momentum $p_{N_{L}}$ of the sterile neutrinos that fly in the direction of the detector, $\int f_{1}\left(p_{N_{L}}\right) d p_{N_{L}}=1$. We note that, for the sterile neutrino mixing with a tau neutrino, a "three stage" processes become important where heavy meson decays produce tauons, and the sterile neutrino is produced in tauon decays. We discuss this case in more detail in Appendix B 2.

The last distribution we need is the distribution $f_{2}\left(z_{H}, p_{N_{L}}\right)$ of the kaon longitudinal travel distance $z_{H}$, $\int f_{2}\left(z_{H}, p_{N_{L}}\right) d z_{H}=1$, which has to be taken into consideration for the sterile neutrinos produced in the kaon decays.

In the experiment, the total number of produced sterile neutrinos $N_{N}$ depends on the number of mesons of each type $N_{H}$ [see Eq. (6)] produced at the target and the probability of their producing the sterile neutrino $\operatorname{Br}(H \rightarrow N \ldots)$ (see Appendix B 2). It can be written as

$N_{N}=N_{\text {POT }} \times \sum_{q, H} M_{p p}(H) \chi_{q} \operatorname{Br}(q \rightarrow H) \operatorname{Br}(H \rightarrow N \ldots)$.

The probability of sterile neutrino decay during the time $t_{N}$ after its production in the sterile neutrino rest frame is described by the usual law (19), where the meson $H$ is replaced by sterile neutrino $N$ :

$$
P\left(t_{N}\right)=1-\exp \left(-\frac{t_{N}}{\tau_{N}}\right)
$$

here $\tau_{N}=\frac{1}{\sum \Gamma(N \rightarrow \ldots)}$ is the sterile neutrino lifetime [see Eqs. (B1)-(B7) in Appendix B 1]. If one wants to use any additional cuts that consider only specific channels, one would have to take into account only these channels. We list some remarks on this possibility in Sec. V.

In the laboratory frame, the sterile neutrino with mass $M_{N}$ and longitudinal momentum $p_{N_{L}}$ travels the distance $l_{N}=\frac{p_{N_{L}}}{M_{N}} t_{N}$ along the beam line before decaying. We note that for the kaons we also have to take into account the distance $z_{H}=\frac{p_{H_{L}}}{M_{H}} t_{H}$ they travel in the decay pipe before decaying. The probability of the sterile neutrino decaying into the visible modes in the interval $L<z_{H}+l_{N}<L+$ $\Delta l$ is

$$
\begin{aligned}
P(L & \left.<z_{H}+l_{N}<L+\Delta l\right) \\
& =\exp \left(-\frac{L-z_{H}}{\tau_{N}} \frac{M_{N}}{p_{N_{L}}}\right)\left(1-\exp \left(-\frac{\Delta l}{\tau_{N}^{\prime}} \frac{M_{N}}{p_{N_{L}}}\right)\right) .
\end{aligned}
$$

Here $\left(\tau_{N}^{\prime}\right)^{-1}$ is the sum of all detectable (visible) sterile neutrino decay modes (B1)-(B7), i.e., all modes besides the three neutrino decay channel (B5). If one needs to take into account only some specific decay modes-for example, to 
apply some background cut-one has to consider only these modes in $\left(\tau_{N}^{\prime}\right)^{-1}$.

To account for the distribution of the sterile neutrino momentum $p_{N_{L}}$ and the distribution of the kaon longitudinal travel distance $z_{H}$, the resulting value of the sterile neutrino probability of decaying in the detector volume reads

$$
\begin{aligned}
P= & \int_{0}^{p_{N_{L}}^{\max }} d p_{N_{L}} f_{1}\left(p_{N_{L}}\right) \\
& \times \int_{0}^{l_{\text {decay pipe }}} d z_{H} f_{2}\left(z_{H}, p_{N_{L}}\right) P\left(L<z_{H}+l_{N}<L+\Delta l\right) .
\end{aligned}
$$

The number of sterile neutrinos $N_{\text {detector }}$ that decay with the probability $P$ inside the detector of the length $\Delta l$ can be expressed as

$$
N_{\text {detector }}=N_{N} \cdot \zeta_{N} \cdot P,
$$

where $N_{N}$ is the total number of produced sterile neutrinos and $\zeta_{N}$ is the portion that flies toward the detector. For a sterile neutrino with fixed mass and mixing, Eq. (26) gives us the total number of sterile neutrino decays inside the detector volume. Sterile neutrino lifetime depends on the mixing as $\tau_{N}=T_{N}|U|^{-2}$, where the numerical coefficient $T_{N}$ does not depend on $|U|^{2}$ [see Eqs. (B1)-(B7) in Appendix B 1]. In the same way one obtains $\tau_{N}^{\prime}=T_{N}^{\prime}|U|^{-2}$, and the numerical coefficient $T_{N}^{\prime}$ does not depend on $|U|^{2}$. For the number of produced sterile neutrinos, we have $N_{N}=\mathcal{N}_{N}|U|^{2}$, where the numerical coefficient $\mathcal{N}_{N}$ does not depend on $|U|^{2}$ [see Eq. (21) and Eqs. (B8), (B9), (B15), and (B17)-(B19) in Appendix B 2]. Therefore the isocontour for a specific value $N_{\text {detector }}$ consists of the values $M_{N},|U|^{2}$ obeying the equation

$$
\begin{aligned}
N_{\text {detector }}= & |U|^{2} \mathcal{N}_{N} \zeta_{N} \int_{0}^{p_{N_{L}}^{\max }} d p_{N_{L}} f_{1}\left(p_{N_{L}}\right) \\
& \times \int_{0}^{l_{\text {decaypipe }}} d z_{H} f_{2}\left(z_{H}, p_{N_{L}}\right) \exp \left(-\frac{L-z_{H}}{T_{N}} \frac{M_{N}}{p_{N_{L}}}|U|^{2}\right) \\
& \times\left(1-\exp \left(-\frac{\Delta l}{T_{N}^{\prime}} \frac{M_{N}}{p_{N_{L}}}|U|^{2}\right)\right) .
\end{aligned}
$$

When the value of the sterile neutrino lifetime satisfies $\tau_{N} \gg\left(L-z_{H}\right) \frac{M_{N}}{p_{N_{L}}}$, Eq. (23) has a simple limit:

$$
P_{1}\left(L<z_{H}+l_{N}<L+\Delta l\right) \approx \frac{\Delta l}{T_{N}^{\prime}} \frac{M_{N}}{p_{N_{L}}}|U|^{2} .
$$

In that case, almost none of the produced sterile neutrinos decay before they reach the detector, and only a small portion of them decay in the detector volume. From Eq. (26), we obtain $N_{\text {detector }}=I \times|U|^{4}$, where the numerical coefficient $I$ does not depend on $|U|^{2}$. Therefore, for an isocontour for a value $N_{\text {detector }}$, if $|U|^{2} \ll \frac{T_{N}}{L-z_{H}} \frac{p_{N_{L}}}{M_{N}}$, Eq. (26) can be rewritten in a simple form:

$$
|U|^{2}=\sqrt{\frac{N_{\text {detector }}}{I}} .
$$

The second important approximation of Eq. (23) is obtained for $\tau_{N}^{\prime} \ll \Delta l \frac{M_{N}}{p_{N_{L}}}$ :

$$
P_{2}\left(L<z_{H}+l_{N}<L+\Delta l\right) \approx \exp \left(-\frac{L-z_{H}}{\tau_{N}} \frac{M_{N}}{p_{N_{L}}}\right) .
$$

In that case, almost all produced sterile neutrinos decay before they reach the detector, and almost all of the sterile neutrinos that reached the detector decay inside the detector volume. Therefore the isocontour for $N_{\text {detector }}$ consists of the values $M_{N},|U|^{2}$ that satisfy the somewhat easier equation

$$
\begin{aligned}
N_{\text {detector }}= & |U|^{2} \mathcal{N}_{N} \zeta_{N} \int_{0}^{p_{N_{L}}^{\max }} d p_{N_{L}} f_{1}\left(p_{N_{L}}\right) \\
& \times \int_{0}^{l_{\text {decay pipe }}} d z_{H} f_{2}\left(z_{H}, p_{N_{L}}\right) \\
& \times \exp \left(-\frac{L-z_{H}}{T_{N}} \frac{M_{N}}{p_{N_{L}}}|U|^{2}\right) .
\end{aligned}
$$

For a fixed value of $M_{N}$, the resulting isocontours consist of two values of $|U|^{2}$ which, for most of the considered mass range, satisfy the conditions of these two approximations. Starting with some value of $M_{N}$, they are no more viable, and one has to use Eq. (26) as it is.

\section{RESULTS}

In this section, we present our isocontours and analysis. We take the beam properties and geometrical sizes as they are described in Refs. [17-20]: $N_{P O T}=1.1 \times 10^{22}$ (this corresponds to the total expected number of protons on target over ten years), $\Delta h=3.5 \mathrm{~m}, L=574 \mathrm{~m}$, $l_{\text {decay pipe }}=194 \mathrm{~m}, r_{\text {decay pipe }}=2 \mathrm{~m}$; see Tables I and II.

For simplicity, in our analysis we vary only two parameters: the detector effective length $\Delta l$ and the number $N_{\text {detector }}$ of the sterile neutrino decays inside the detector volume. In the ideal situation in which there is absolutely no background, according to the Poisson distribution, $N_{\text {detector }}=3$ should be enough to place the limit at 95\% C.L. to announce the discovery of the heavy sterile neutrinos. Unfortunately, the absence of an active neutrino event background is not a realistic assumption for the DUNE Near Detector. The main goal of the Near Detector is to characterize the beam of active neutrinos. Therefore it will be designed in such a way as to increase the probability of neutrino interactions inside the detector. From the point 


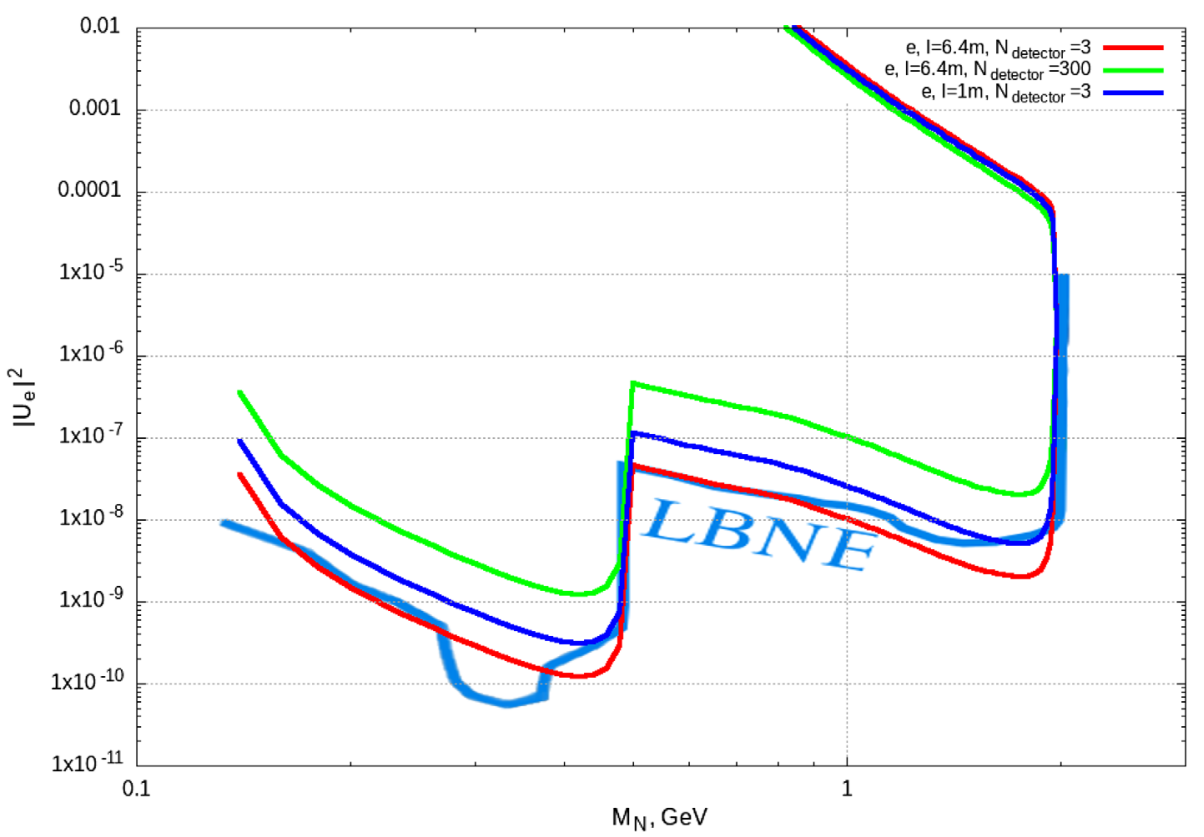

FIG. 2. The number of expected sterile neutrino decays in the detector volume in the plane $M_{N}-|U|^{2}$ for the case of mixing with the electron neutrino. The LBNE (steel blue) line is a previous sensitivity estimate [23]. The red line is our estimate for $\Delta l=6.4 \mathrm{~m}$ and $N_{\text {detector }}=3$, the blue line is for $\Delta l=1.0 \mathrm{~m}$ and $N_{\text {detector }}=3$, and the green line is for $\Delta l=6.4 \mathrm{~m}$ and $N_{\text {detector }}=300$.

of view of a sterile neutrino search, such interactions would serve as a background. We avoid all of the issues of the experimental detection efficiencies and reconstruction effects by presenting only the isocontours for the number of expected heavy neutrino decays into visible modes inside the detector volume, in the plane $M_{N}-|U|^{2}$.
We present our results for mixing with the electron, muon, and tau neutrino in Figs. 2-4, respectively. For the red lines, we take $N_{\text {detector }}=3$, and for the green lines, we take $N_{\text {detector }}=300$. For these lines, we take the currently considered detector length $\Delta l=6.4 \mathrm{~m}$ from Ref. [20]. All isocontours are calculated in accordance with Eq. (26).

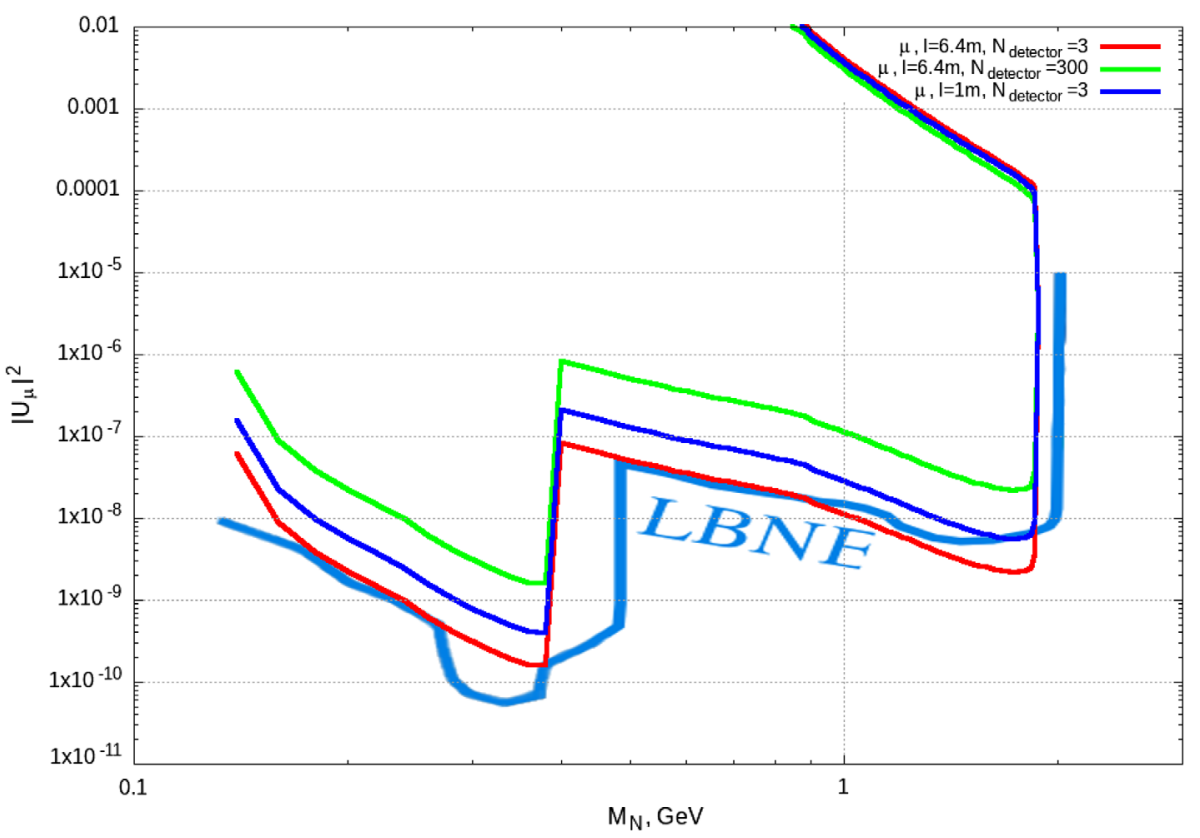

FIG. 3. The number of expected sterile neutrino decays in the detector volume in the plane $M_{N}-|U|^{2}$ for the case of mixing with the muon neutrino. The LBNE (steel blue) line is a previous sensitivity estimate [23]. The red line is our estimate for $\Delta l=6.4 \mathrm{~m}$ and $N_{\text {detector }}=3$, the blue line is for $\Delta l=1.0 \mathrm{~m}$ and $N_{\text {detector }}=3$, and the green line is for $\Delta l=6.4 \mathrm{~m}$ and $N_{\text {detector }}=300$. 


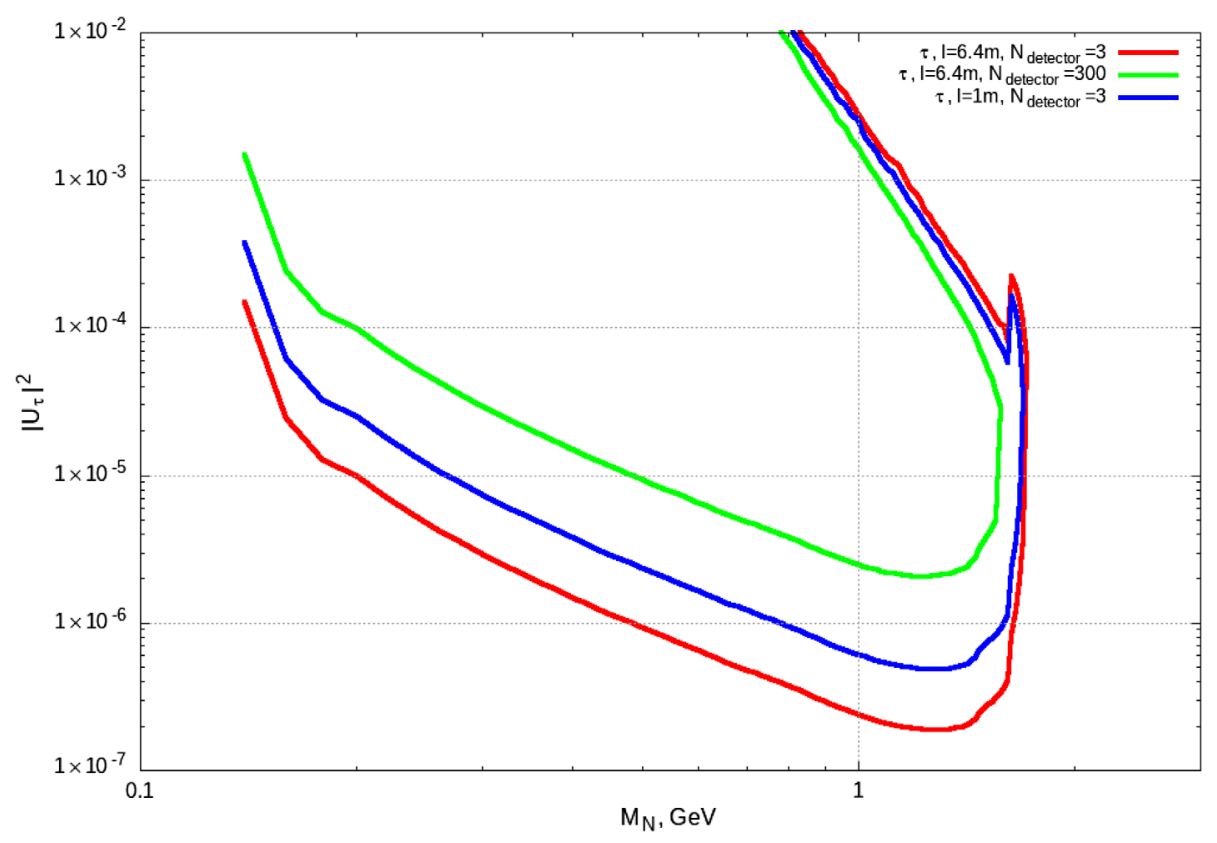

FIG. 4. The number of expected sterile neutrino decays in the detector volume in the plane $M_{N}-|U|^{2}$ for the case of mixing with the tauon neutrino. The red line is our estimate for $\Delta l=6.4 \mathrm{~m}$ and $N_{\text {detector }}=3$, the blue line is for $\Delta l=1.0 \mathrm{~m}$ and $N_{\text {detector }}=3$, and the green line is for $\Delta l=6.4 \mathrm{~m}$ and $N_{\text {detector }}=300$. The small feature at $M_{N}=1.63 \mathrm{GeV}$ is due to the disappearance of the channel $\tau \rightarrow \pi N$, which is dominating up to that point.

There is another possibility that we point out. If we had additional free space in front of the main detector, we could place there a small additional detector, sensitive to sterile neutrino decays. To reduce the active neutrino event background, as well as to minimize the effect of the additional detector on active neutrino study, the additional detector should be almost empty inside. That would allow for detecting of the sterile neutrino decays in this empty space, where there are few active neutrino interactions. Depending on the design, it could provide better sensitivity to mixing with active neutrinos than the main detector. In Figs. 2-4, we show that case with blue lines, for which we take $\Delta l=1 \mathrm{~m}$ and $N_{\text {detector }}=3$. From these figures, one can see that, depending on its configuration, a small additional detector with good efficiency could provide better limits than the main detector, which is overburdened with the active neutrino background.

For reference, we present in Figs. 2 and 3 the estimate from the LBNE design report [23]. That estimate was made simply by rescaling the CHARM [4] and CERN PS191 [6] results, taking into account the relevant proton beam and detector geometry parameters of LBNE and CHARM and PS191 experiments. The length of the LBNE Near Detector was taken to be $\Delta l=30 \mathrm{~m}$. These lines were calculated for the case in which a sterile neutrino mixes with every type of active neutrino, while we present the mixing with a specific type. This results in a difference in the shape of the curve. Because of the difference in mass between the electron and muon, decays of kaons into the sterile neutrino stop at lower masses of the sterile neutrino $M_{N}$ for a mixing with a muon neutrino than for a mixing with an electron neutrino. In Figs. 2 and 3, one can see these steps at $M_{N} \sim M_{K}-m_{e}$ and $M_{N} \sim M_{K}-m_{\mu}$, respectively. For the LBNE line, this shift occurs in two steps, as the muon part of the mixing disappears at a lower sterile neutrino mass than the electron part. We did not find estimates for LBNE limits on a mixing with a tau neutrino, so in Fig. 4 we present only our estimates.

One can find current limits on the mixing (and some predictions) in Refs. [13-15,30,31].

\section{THE VALIDITY OF THE RESULTS}

In this section, we estimate the active neutrino flux as a validation of our results and present some ideas on the scale of the signal to background ratio in the sterile neutrino search and how it can be improved.

We have performed a special simulation of the flux of $M_{N}=0.01 \mathrm{eV}$ sterile neutrinos through the Far Detector with $N_{P O T}=10^{20} s$ to check our assumptions of the meson production and decay and compare them with the results from Ref. [18]. We compute the number of "neutrinos" reaching the Far Detector for $N_{P O T}=10^{20}$ and divide by the detectors' frontal area and the energy of the neutrino. The basic idea is that the obtained results should more or less correspond to active neutrino fluxes from pion and kaon decays. For that purpose, we consider for the "muon neutrino" $N_{\mu}$ the following processes: $\pi^{+} \rightarrow \mu^{+} N_{\mu}$, $K^{+} \rightarrow \mu^{+} N_{\mu}, K^{+} \rightarrow \pi^{0} \mu^{+} N_{\mu}, K^{0} \rightarrow \pi^{-} \mu^{+} N_{\mu}$ as well as the decay $\mu^{+} \rightarrow e^{+} N_{e} N_{\mu}$ for muons produced in these 
processes; for the number of "electron neutrinos" $N_{e}$ we have the same muon decays and the processes $K^{-} \rightarrow$ $\pi^{0} e^{-} N_{e}, K^{0} \rightarrow \pi^{+} e^{-} N_{e}$. Pion decays and the subsequent muon decays are the most significant contributors to the active neutrino production. In fact, according to our calculations, most of the electronic neutrinos are produced in muon decays.

Note that, for the secondary pions and kaons produced in the target, there exists a nonzero chance to interact with the matter of the target and, effectively, to lose energy in that interaction. The longer the target is, the more significant that possibility becomes. Yet, for short-lived heavy mesons, this effect is insignificant. As sterile neutrinos are mainly produced in the decays of heavy mesons, we do not study the effects of the length of the target at all.

The fluxes of the neutrino and antineutrino are considered to be the same because we do not account for the presence of the horn focusing systems. Horn focusing systems affect all charged particles, but particularly the charged pions and kaons, as they are relatively long-lived. As the name implies, horns would focus these particles, causing more of them to fly in the direction of the detector. Usually, horns are specialized to focus pions, as their flux is much higher than the flux of kaons. The usage of horns in other experiments increased the resulting flux of neutrinos by several times. Recall that, for the sterile neutrino search, active neutrino events serve as a background. It should be compensated for a little because of the focusing of kaons that produce sterile neutrinos, but the overall effect of horns is considered to be negative for the sterile neutrino search.

We present our results, as well as the neutrino fluxes adopted from Ref. [18] in Figs. 5 and 6. As we do not account for the presence of the horn focusing systems, our results should lie between the neutrino and antineutrino curves. In neutrino (antineutrino) mode, the focusing system increases the flux of neutrinos (antineutrinos) but decreases the flux of the antineutrinos (neutrinos). This behavior can be seen in Figs. 5 and 6. Note that neutrinos with higher energy are less affected by horn systems, and results from Ref. [18] for this part of the spectrum are, overall, in good correlation with our estimate. We note that the shape of the resulting energy-flux curve is somewhat different from Ref. [18]. Aside from the presence of the horns the difference can also originate from the more rare processes which are not accounted for in our estimate. The slight discrepancy in the high-energy part should originate from the secondary pion interactions with the target, as mentioned above. Overall, we find these results satisfactory given the crudeness of the estimate and the factor of the presence of the horns.

In the sterile neutrino search, there are several methods that can be used to improve the signal to background ratio that we want to briefly overview here. One such way is to take into consideration only certain decay modes. Among various sterile neutrino decay modes, the most promising

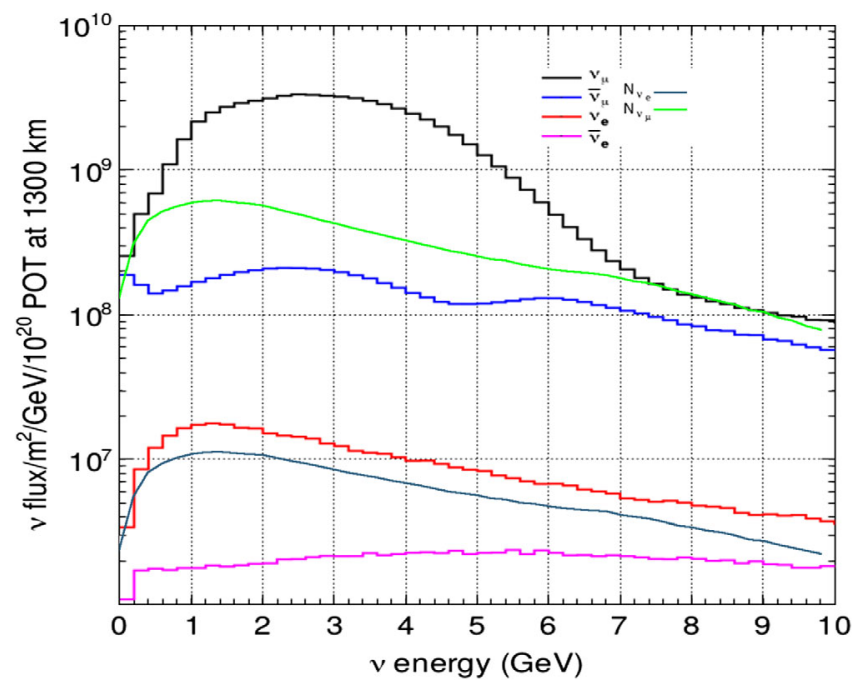

FIG. 5. Neutrino fluxes adopted from Ref. [18] (the black, blue, red, and violet lines) for the neutrino mode, generated with a $120 \mathrm{GeV}$ primary proton beam and our corresponding estimate without the horns (steel blue and green lines).

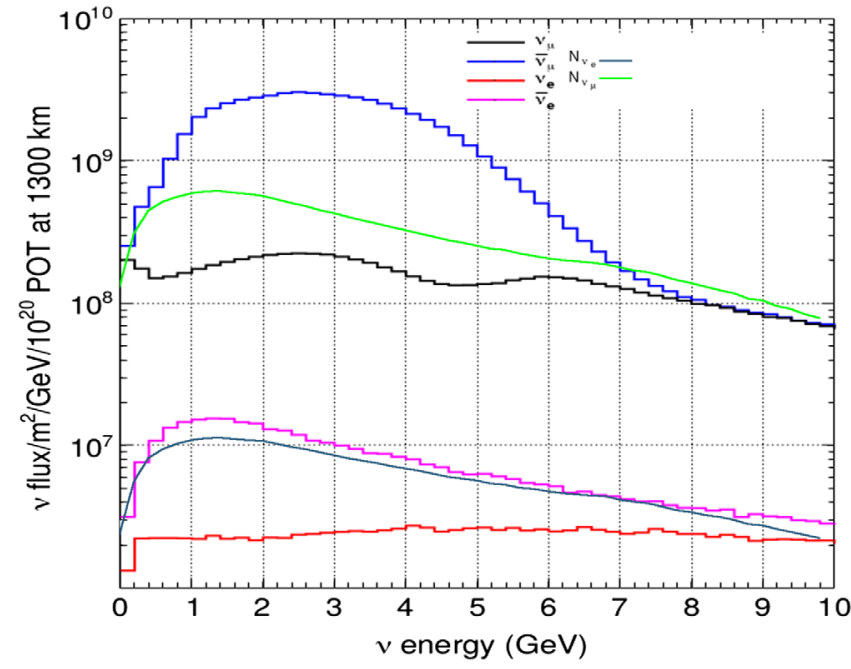

FIG. 6. Neutrino fluxes adopted from Ref. [18] (the black, blue, red, and violet lines) for the antineutrino mode, generated with a $120 \mathrm{GeV}$ primary proton beam and our corresponding estimate without the horns (steel blue and green lines).

ones are the two-body decays. The products of two-body decays have a fixed momentum in the decaying particle rest frame. Its value depends only on the decaying particle mass. This fact allows for a more precise reconstruction of the mass of the decaying particle than in the case of threeor-more-body decays.

Let us consider a specific example: the decay $N \rightarrow \pi^{+} \mu^{-}$. The Monte Carlo simulation results for $N_{P O T}=10^{20}$ presented in Ref. [18] suggest 44000 events of the $\nu_{\mu} X \rightarrow$ $\pi^{+} \mu^{-} X$ type, where $X$ is the atom of the target. Hence for $N_{P O T}=1.1 \times 10^{22}$, adopted in this paper, the number of 
background events integrated over the energy easily reaches $4.8 \times 10^{6}$. For the sterile neutrino two-body decay, however, not all of these events serve as a background. If one reconstructs the energy distribution of the detected $\mu, \pi$, the products of the sterile neutrino decays would form characteristic peaks. Only events for $\mu, \pi$ with the same energy as these peaks would serve as a background for the sterile neutrino signal. Obviously, similar considerations apply to other decay modes as well. Unfortunately, the simulation/ reconstruction of such an energy distribution depends heavily on the detector specifics and is rather hard to perform.

An additional possibility is to account for the fact that sterile neutrinos that reach the detector have very small transversal momentum. Therefore the sum of the resulting particles transversal momentum should also be close to zero. If one cuts all events for which this is not true, one would reduce the amount of background.

Overall, a detailed study of the detector efficiency will be possible only after the final decision on the design of the Near Detector is made.

\section{CONCLUSIONS}

In this paper, we calculated the prospects for the sterile neutrino search in the upcoming experiment DUNE. We present the isocontours for the number of expected heavy neutrino decays inside the detector volume, in the plane $M_{N}-|U|^{2}$. Our more optimistic predictions are approximately of the same order as the previous estimates, while more conservative ones lie higher than it, but still lower than the current limits. We point out that, for the search of sterile neutrinos, an additional small detector that is almost empty inside could provide better sensitivity than the main detector. Another possibility to enhance the signal to background ratio can be a special run with a really short proton spill duration and a lower proton beam energy. Overall, our estimates show that while DUNE's main scientific goal is the measurement of active neutrino parameters, it would still be able to probe a currently unrestricted part of the sterile neutrino parameter space.

\section{ACKNOWLEDGMENTS}

We would like to thank D. Gorbunov and Y. Kudenko for the valuable discussions. This work was supported by RSF Grant No. 17-12-01547.

\section{APPENDIX A: PARAMETERS}

In this section, we list the experimental values of the parameters used in our work.

Lepton masses: $l \in\{e, \mu, \tau\}: M_{e}=0.5109989461 \mathrm{MeV}$, $M_{\mu}=105.6583745 \mathrm{MeV}, M_{\tau}=1.77686 \mathrm{GeV}$ [27].

Tauon average lifetime: $\tau_{\tau}=2.903 \times 10^{-4} \mathrm{~ns}$ [27].

Fermi constant: $G_{F}=1.16637877 \times 10^{-5} \mathrm{GeV}^{-2}$ [27]. Weinberg angle: $\sin ^{2} \theta_{W}=0.23122$ [27].
TABLE III. Cabibbo-Kobayashi-Maskawa (CKM) matrix elements [27].

\begin{tabular}{lccccc}
\hline \hline$V_{u d}$ & $V_{u s}$ & $V_{c d}$ & $V_{c s}$ & $V_{u b}$ & $V_{c b}$ \\
\hline 0.97420 & 0.2243 & 0.218 & 0.997 & 0.00394 & 0.0422 \\
\hline \hline
\end{tabular}

$\rho$-meson decay constant: $g_{\rho}=0.162 \mathrm{GeV}^{2} \quad$ [29]. Cabibbo-Kobayashi-Maskawa (CKM) matrix elements are presented in Table III [27], and some other relevant meson decay parameters are presented in Table IV [27,29].

\section{Form factors}

The basic formula [29] is

$$
\begin{aligned}
f\left(q^{2}\right)= & \frac{1}{1-q^{2} / M_{\text {pole }}^{2}} \\
& \times \sum_{n=0}^{N-1} a_{n}\left[\left(z\left(q^{2}\right)\right)^{n}-(-1)^{n-N} \frac{n}{N}\left(z\left(q^{2}\right)\right)^{N}\right],
\end{aligned}
$$

where

$$
z\left(q^{2}\right) \equiv \frac{\sqrt{t_{+}-q^{2}}-\sqrt{t_{+}-t_{0}}}{\sqrt{t_{+}-q^{2}}+\sqrt{t_{+}-t_{0}}}
$$

with

$$
\begin{gathered}
t_{+}=\left(m_{H}+m_{H^{\prime}}\right)^{2}, \\
t_{0}=\left(m_{H}+m_{H^{\prime}}\right)\left(\sqrt{m_{H}}-\sqrt{m_{H^{\prime}}}\right)^{2} .
\end{gathered}
$$

TABLE IV. Relevant meson decay parameters [27,29].

\begin{tabular}{lccc}
\hline \hline$H$ & $M_{H}, \mathrm{MeV}[27]$ & $\tau_{H}, \mathrm{~ns}[27]$ & $f_{H}, \mathrm{MeV}[29]$ \\
\hline$\pi^{+}$ & 139.57061 & 26.033 & 130.2 \\
$\pi^{0}$ & 134.977 & $8.52 \mathrm{e}-8$ & 130.2 \\
$K^{+}$ & 493.677 & 12.38 & 155.6 \\
$K_{L}^{0}$ & 497.611 & 51.16 & \\
$K_{S}^{0}$ & 497.611 & 0.089564 & \\
$\eta$ & 547.862 & & 81.7 \\
$\rho$ & 775.26 & & -94.7 \\
$\eta^{\prime}$ & 957.78 & & 212 \\
$D^{+}$ & 1869.65 & $1.04 \mathrm{e}-3$ & \\
$D^{0}$ & 1864.83 & $4.101 \mathrm{e}-4$ & 249 \\
$D_{s}^{+}$ & 1968.34 & $5.04 \mathrm{e}-4$ & 187 \\
$B^{+}$ & 5279.32 & $1.638 \mathrm{e}-3$ & \\
$B^{0}$ & 5279.63 & $1.52 \mathrm{e}-3$ & \\
$B_{s}^{0}$ & 5366.89 & $1.509 \mathrm{e}-3$ & 434 \\
$B_{c}^{+}$ & 6274.9 & $5.07 \mathrm{e}-4$ & \\
\hline \hline
\end{tabular}


TABLE V. Best fit parameters [29] for the form factors of the $K \rightarrow \pi$ transition.

\begin{tabular}{lccc}
\hline \hline$H, H^{\prime}$ & $f_{+, 0}(0)$ & $\lambda_{+}$ & $\lambda_{0}$ \\
\hline$K^{0}, \pi^{+}$ & 0.970 & 0.0267 & 0.0117 \\
$K^{+}, \pi^{0}$ & 0.970 & 0.0277 & 0.0183 \\
\hline \hline
\end{tabular}

\section{a. K-meson form factors}

Form factors of the $K \rightarrow \pi$ transition are well described by the linear approximation

$$
f_{+, 0}^{K \pi}\left(q^{2}\right)=f_{+, 0}^{K \pi}(0)\left(1+\lambda_{+, 0} \frac{q^{2}}{m_{\pi^{+}}^{2}}\right) .
$$

Best fit parameters are presented in Table V [29].

\section{b. D-meson form factors}

The form factors of $D \rightarrow K, \pi$ are

$$
f\left(q^{2}\right)=\frac{f(0)-c\left(z\left(q^{2}\right)-z(0)\right)\left(1+\frac{z\left(q^{2}\right)+z(0)}{2}\right)}{1-P q^{2}} .
$$

The form factors of $D \rightarrow \eta$ are

$$
f_{+}^{D_{s} \eta}\left(q^{2}\right)=\frac{f_{+}^{D_{s} \eta}(0)}{\left(1-\frac{q^{2}}{M_{D_{s}^{*}}^{2}}\right)\left(1-\alpha_{+}^{D_{s} \eta} \frac{q^{2}}{M_{D_{s}^{*}}^{2}}\right)},
$$

where $f_{+}^{D_{s} \eta}(0)=0.495, M_{D_{s}^{*}}=2.112, \alpha_{+}^{D_{s} \eta}=0.198$ [29],

$$
f_{0}^{D_{s} \eta}\left(q^{2}\right)=\frac{f_{0}^{D_{s} \eta}(0)}{1-\alpha_{0}^{D_{s} \eta} \frac{q^{2}}{M_{D_{s}^{*}}^{2}}}
$$

$f_{0}^{D_{s} \eta}\left(q^{2}\right)$ is not well constrained by the experimental data, so we take $f_{0}^{D_{s} \eta}(0)=f_{+}^{D_{s} \eta}(0)$ and $\alpha_{0}^{D_{s} \eta}=0$ [29].

Best fit parameters are presented in Table VI [29].

\section{c. B-meson form factors}

Form factors of $B \rightarrow D, \pi$ are described by the basic formula (A1). Best fit parameters are presented in Table VII [29].

TABLE VI. Best fit parameters [29] for the form factors of the $D \rightarrow K, \pi$ transition.

\begin{tabular}{lccc}
\hline \hline$f$ & $f(0)$ & $\mathrm{c}$ & $P\left(\mathrm{GeV}^{-} 2\right)$ \\
\hline$f_{+}^{D K}$ & 0.7647 & 0.066 & 0.224 \\
$f_{0}^{D K}$ & 0.7647 & 2.084 & 0 \\
$f_{+}^{D \pi}$ & 0.6117 & 1.985 & 0.1314 \\
$f_{0}^{D \pi}$ & 0.6117 & 1.188 & 0.0342 \\
\hline \hline
\end{tabular}

TABLE VII. Best fit parameters [29] for the form factors of the $B \rightarrow D, \pi$ transition.

\begin{tabular}{lcccc}
\hline \hline$f$ & $M_{\text {pole }} \mathrm{GeV}$ & $a_{0}$ & $a_{1}$ & $a_{2}$ \\
\hline$f_{+}^{B_{(s)} D_{(s)}}$ & $\infty$ & 0.909 & -7.11 & 66 \\
$f_{0}^{B_{(s)} D_{(s)}}$ & $\infty$ & 0.794 & -2.45 & 33 \\
$f_{+}^{B K}$ & 5.325 & 0.360 & -0.828 & 1.1 \\
$f_{0}^{B K}$ & 5.65 & 0.233 & 0.197 & 0.18 \\
$f_{+}^{B \pi}$ & 5.325 & 0.404 & -0.68 & -0.86 \\
$f_{0}^{B \pi}$ & 5.65 & 0.490 & -1.61 & 0.93 \\
\hline \hline
\end{tabular}

\section{d. Meson form factors for decay into vector meson}

Standard axial form factors $A_{0}\left(q^{2}\right), A_{1}\left(q^{2}\right), A_{2}\left(q^{2}\right)$ and vector form factor $V\left(q^{2}\right)$ can be parametrized as

$$
\begin{gathered}
V\left(q^{2}\right)=\frac{f_{V}^{h h^{\prime}}}{\left(1-\frac{q^{2}}{M_{V}^{h^{2}}}\right)\left(1-\sigma_{V}^{h h^{\prime}} \frac{q^{2}}{M_{V}^{h^{2}}}-\zeta_{V}^{h h^{\prime}} \frac{q^{4}}{M_{V}^{h^{4}}}\right)}, \\
A_{0}\left(q^{2}\right)=\frac{f_{A_{0}}^{h h^{\prime}}}{\left(1-\frac{q^{2}}{M_{P}^{h^{2}}}\right)\left(1-\sigma_{A_{0}}^{h h^{\prime}} \frac{q^{2}}{M_{V}^{h^{2}}}-\zeta_{A_{0}}^{h h^{\prime}} \frac{q^{4}}{M_{V}^{h^{4}}}\right)}, \\
A_{1,2}\left(q^{2}\right)=\frac{f_{A_{1,2}}^{h h^{\prime}}}{1-\sigma_{A_{1,2}}^{h h^{\prime}} \frac{q^{2}}{M_{V}^{h^{2}}}-\zeta_{A_{1,2}}^{h h^{\prime}} \frac{q^{4}}{M_{V}^{h^{4}}}} .
\end{gathered}
$$

Best fit parameters are presented in Table VIII [29]

TABLE VIII. Best fit parameters [29] of the meson form factors of the decays into the vector meson.

\begin{tabular}{llllll}
\hline \hline$h h^{\prime}$ & $D K^{*}$ & $B D^{*}$ & \multicolumn{1}{c}{$B \rho$} & $B_{s} D^{*}$ & $B_{s} K$ \\
\hline$f_{V}^{h h^{\prime}}$ & 1.03 & 0.76 & 0.295 & 0.95 & 0.291 \\
$f_{A_{0}}^{h h^{\prime}}$ & 0.76 & 0.69 & 0.231 & 0.67 & 0.289 \\
$f_{A_{1}}^{h h^{\prime}}$ & 0.66 & 0.66 & 0.269 & 0.70 & 0.287 \\
$f_{A_{2}}^{h h^{\prime}}$ & 0.49 & 0.62 & 0.282 & 0.75 & 0.286 \\
$\sigma_{V}^{h h^{\prime}}$ & 0.27 & 0.57 & 0.875 & 0.372 & -0.516 \\
$\sigma_{A_{0}}^{h h^{\prime}}$ & 0.17 & 0.59 & 0.796 & 0.350 & -0.383 \\
$\sigma_{A_{1}}^{h h^{\prime}}$ & 0.3 & 0.78 & 0.54 & 0.463 & 0 \\
$\sigma_{A_{2}}^{h h^{\prime}}$ & 0.67 & 1.4 & 1.34 & 1.04 & 1.05 \\
$\zeta_{V}^{h h^{\prime}}$ & 0 & 0 & 0 & 0.561 & 2.10 \\
$\zeta_{A_{0}}^{h h^{\prime}}$ & 0 & 0 & 0.055 & 0.600 & 1.58 \\
$\zeta_{A_{1}}^{h h^{\prime}}$ & 0.2 & 0 & 0 & 0.510 & 1.06 \\
$\zeta_{A_{2}}^{h h^{\prime}}$ & 0.16 & 0.41 & -0.21 & 0.070 & -0.074 \\
$M_{P}^{h}(\mathrm{GeV})$ & 1.969 & 6.275 & 5.279 & 6.275 & 5.367 \\
$M_{V}^{h}(\mathrm{GeV})$ & 2.112 & 6.331 & 5.325 & 6.331 & 5.415 \\
\hline \hline
\end{tabular}




\section{APPENDIX B: FORMULAS}

\section{Sterile neutrino decays}

The two-particle sterile neutrino decays [21,29] are

$$
\begin{gathered}
\Gamma\left(N \rightarrow H^{0} \nu_{\alpha}\right)=\frac{\left|U_{\alpha}\right|^{2}}{32 \pi} G_{F}^{2} f_{H^{0}}^{2} M_{N}^{3}\left(1-\frac{M_{H^{0}}^{2}}{M_{N}^{2}}\right)^{2} \\
\Gamma\left(N \rightarrow H^{+} l_{\alpha}^{-}\right)=\frac{\left|U_{\alpha}\right|^{2}}{16 \pi} G_{F}^{2}\left|V_{H}\right|^{2} f_{H}^{2} M_{N}^{3}\left(\left(1-\frac{M_{l}^{2}}{M_{N}^{2}}\right)^{2}-\frac{M_{H}^{2}}{M_{N}^{2}}\left(1+\frac{M_{l}^{2}}{M_{N}^{2}}\right)\right) \\
\times \sqrt{\left(1-\frac{\left(M_{H}-M_{l}\right)^{2}}{M_{N}^{2}}\right)\left(1-\frac{\left(M_{H}+M_{l}\right)^{2}}{M_{N}^{2}}\right)}, \\
\Gamma\left(N \rightarrow V^{+} l_{\alpha}^{-}\right)=\frac{\left|U_{\alpha}\right|^{2}}{16 \pi} \frac{g_{V^{+}}^{2}}{M_{V^{+}}^{2}} G_{F}^{2}\left|V_{V}\right|^{2} M_{N}^{3}\left(\left(1-\frac{M_{l}^{2}}{M_{N}^{2}}\right)^{2}+\frac{M_{V^{+}}^{2}}{M_{N}^{2}}\left(1+\frac{\left.M_{l}^{2}-2 M_{V^{+}}^{2}\right)}{M_{N}^{2}}\right)\right) \\
\times \sqrt{\left(1-\frac{\left(M_{V^{+}}-M_{l}\right)^{2}}{M_{N}^{2}}\right)\left(1-\frac{\left(M_{V^{+}}+M_{l}\right)^{2}}{M_{N}^{2}}\right)} \\
\Gamma\left(N \rightarrow V^{0} \nu_{\alpha}\right)=\frac{\left|U_{\alpha}\right|^{2}}{32 \pi} \frac{g_{V^{0}}^{2} k_{V}^{2}}{M_{V^{0}}^{2}} G_{F}^{2} M_{N}^{3}\left(1+2 \frac{M_{V^{0}}^{2}}{M_{N}^{2}}\right)\left(1-\frac{M_{V^{0}}^{2}}{M_{N}^{2}}\right)^{2},
\end{gathered}
$$

where $H^{0} \in\left\{\pi^{0}, \eta, \eta^{\prime}, \ldots\right\}, H^{+} \in\left\{\pi^{+}, K^{+}, D^{+}, \ldots\right\}, V^{0} \in\left\{\rho^{0}, \ldots\right\}, V^{+} \in\left\{\rho^{+}, \ldots\right\}$ (see Table IV). In this work, we use only $k_{\rho}=1-2 \sin ^{2} \theta_{W}$.

The three-particle sterile neutrino decays [21,29] are

$$
\begin{gathered}
\Gamma\left(N \rightarrow \nu_{\alpha} \sum_{\beta} \bar{\nu}_{\beta} \nu_{\beta}\right)=\frac{G_{F}^{2} M_{N}^{5}}{192 \pi^{3}}\left|U_{\alpha}\right|^{2}, \\
\Gamma\left(N \rightarrow l_{\alpha \neq \beta}^{-} l_{\beta}^{+} \nu_{\beta}\right)=\frac{G_{F}^{2} M_{N}^{5}\left|U_{\alpha}\right|^{2}\left(1-8 x_{l}^{2}+8 x_{l}^{6}-x_{l}^{8}-12 x_{l}^{4} \log x_{l}^{2}\right),}{192 \pi^{3}} \\
x_{l}=\frac{\max \left[M_{l_{\alpha}}, M_{l_{\beta}}\right]}{M_{N}},
\end{gathered}
$$

$$
\begin{aligned}
\Gamma\left(N \rightarrow \nu_{\alpha} l_{\beta}^{-} l_{\beta}^{+}\right)= & \frac{G_{F}^{2} M_{N}^{5}}{192 \pi^{3}}\left|U_{\alpha}\right|^{2}\left[\left(C_{1}\left(1-\delta_{\alpha \beta}\right)+C_{3} \delta_{\alpha \beta}\right)\left(\left(1-14 x_{l}^{2}-2 x_{l}^{4}-12 x_{l}^{6}\right) \sqrt{1-4 x_{l}^{2}}+12 x_{l}^{4}\left(x_{l}^{4}-1\right) L\right)\right. \\
& \left.+4\left(C_{2}\left(1-\delta_{\alpha \beta}\right)+C_{4} \delta_{\alpha \beta}\right) \times\left(x_{l}^{2}\left(2+10 x_{l}^{2}-12 x_{l}^{4}\right) \sqrt{1-4 x_{l}^{2}}+6 x_{l}^{4}\left(1-2 x_{l}^{2}+2 x_{l}^{4}\right) L\right)\right],
\end{aligned}
$$

where

$$
L=\log \left[\frac{1-3 x_{l}^{2}-\left(1-x_{l}^{2}\right) \sqrt{1-4 x_{l}^{2}}}{x_{l}^{2}\left(1+\sqrt{1-4 x_{l}^{2}}\right)}\right], \quad x_{l} \equiv \frac{M_{l}}{M_{N}},
$$

and

$$
\begin{array}{ll}
C_{1}=\frac{1}{4}\left(1-4 \sin ^{2} \theta_{w}+8 \sin ^{4} \theta_{w}\right), & C_{2}=\frac{1}{2} \sin ^{2} \theta_{w}\left(2 \sin ^{2} \theta_{w}-1\right), \\
C_{3}=\frac{1}{4}\left(1+4 \sin ^{2} \theta_{w}+8 \sin ^{4} \theta_{w}\right), & C_{4}=\frac{1}{2} \sin ^{2} \theta_{w}\left(2 \sin ^{2} \theta_{w}+1\right) .
\end{array}
$$




\section{Sterile neutrino production}

The two-body meson decays with sterile neutrino production [21] are

$$
\begin{aligned}
\frac{d \operatorname{Br}\left(H^{+} \rightarrow l_{\alpha}^{+} N\right)}{d E_{N}}= & \tau_{H} \frac{G_{F}^{2} M_{N}^{2} M_{H} f_{H}^{2}}{8 \pi}\left|V_{H}\right|^{2}\left|U_{\alpha}\right|^{2}\left(1-\frac{M_{N}^{2}}{M_{H}^{2}}+2 \frac{m_{l}^{2}}{M_{H}^{2}}+\frac{m_{l}^{2}}{M_{N}^{2}}\left(1-\frac{m_{l}^{2}}{M_{H}^{2}}\right)\right) \sqrt{\left(1+\frac{M_{N}^{2}}{M_{H}^{2}}-\frac{m_{l}^{2}}{M_{H}^{2}}\right)^{2}-4 \frac{M_{N}^{2}}{M_{H}^{2}}} \\
& \times \delta\left(E_{N}-\frac{M_{H}^{2}-M_{l}^{2}+M_{N}^{2}}{2 M_{H}}\right) .
\end{aligned}
$$

We take the following decays into two particles: $K^{+} \rightarrow l^{+} N, D^{+} \rightarrow l^{+} N, D_{s}^{+} \rightarrow l^{+} N, B^{+} \rightarrow l^{+} N, B_{c}^{+} \rightarrow l^{+} N$. Twoparticle meson decay CKM matrix elements are presented in Table IX.

The three-particle scalar meson decays with sterile neutrino production [21] are

$$
\begin{aligned}
\frac{d \operatorname{Br}\left(H \rightarrow H^{\prime} l_{\alpha} N\right)}{d E_{N}}= & \tau_{H}\left|U_{\alpha}\right|^{2} C_{K}^{2} \frac{G_{F}^{2}\left|V_{H H^{\prime}}\right|^{2}}{64 \pi^{3} M_{H}^{2}} \int_{q_{\min }^{2}}^{q_{\max }^{2}} d q^{2}\left(f_{-}^{2}\left(q^{2}\right)\left(q^{2}\left(M_{N}^{2}+M_{l}^{2}\right)-\left(M_{N}^{2}-M_{l}^{2}\right)^{2}\right)\right. \\
& +2 f_{-}\left(q^{2}\right) f_{+}\left(q^{2}\right)\left(M_{N}^{2}\left(2 M_{H}^{2}-2 M_{H^{\prime}}^{2}-4 E_{N} M_{H}-M_{l}^{2}+M_{N}^{2}+q^{2}\right)+M_{l}^{2}\left(4 E_{N} M_{H}+M_{l}^{2}-M_{N}^{2}-q^{2}\right)\right) \\
& +f_{+}^{2}\left(q^{2}\right)\left(\left(4 E_{N} M_{H}+M_{l}^{2}-M_{N}^{2}-q^{2}\right)\left(2 M_{H}^{2}-2 M_{H^{\prime}}^{2}-4 E_{N} M_{H}-M_{l}^{2}+M_{N}^{2}+q^{2}\right)\right. \\
& \left.\left.-\left(2 M_{H}^{2}+2 M_{H^{\prime}}^{2}-q^{2}\right)\left(q^{2}-M_{N}^{2}-M_{l}^{2}\right)\right)\right),
\end{aligned}
$$

where $C_{K}=\frac{1}{\sqrt{2}}$ for $H^{\prime}=\pi^{0}, C_{K}=1$ for all other cases [29] and the $q^{2}$ range is [27]

$$
\begin{gathered}
q_{\min }^{2}=\left(E_{2}^{*}+E_{3}^{*}\right)^{2}-\left(\sqrt{E_{2}^{* 2}-M_{l}^{2}}+\sqrt{E_{3}^{* 2}-M_{N}^{2}}\right)^{2}, \\
q_{\max }^{2}=\left(E_{2}^{*}+E_{3}^{*}\right)^{2}-\left(\sqrt{E_{2}^{* 2}-M_{l}^{2}}-\sqrt{E_{3}^{* 2}-M_{N}^{2}}\right)^{2}, \\
E_{2}^{*}=\frac{M_{H}^{2}+M_{N}^{2}+M_{l}^{2}-M_{H^{\prime}}^{2}-2 M_{H} E_{N}}{2 m_{12}}, \\
E_{3}^{*}=\frac{M_{H} E_{N}-M_{N}^{2}}{m_{12}}, \\
m_{12}=\sqrt{M_{H}^{2}+M_{N}^{2}-2 M_{H} E_{N}} ;
\end{gathered}
$$

the $q^{2}$ range depends on $E_{N}$. Reference [27] provides us with the $E_{N}$ range $\left(M_{H^{\prime}}+M_{l}\right)^{2} \leq m_{12}^{2} \leq\left(M_{H}-M_{N}\right)^{2}$. It is equivalent to $M_{N} \leq E_{N} \leq \frac{1}{2 M_{H}}\left(M_{H}^{2}+M_{N}^{2}-\left(M_{H^{\prime}}+M_{l}\right)^{2}\right)$. Three-particle meson decay CKM matrix elements are presented in Table X.

TABLE IX. Two-particle meson decay CKM matrix elements.

\begin{tabular}{ccccccc}
\hline \hline & $\pi^{+} \rightarrow l^{+} N$ & $K^{+} \rightarrow l^{+} N$ & $D^{+} \rightarrow l^{+} N$ & $D_{s}^{+} \rightarrow l^{+} N$ & $B^{+} \rightarrow l^{+} N$ & $B_{c}^{+} \rightarrow l^{+} N$ \\
\hline$V_{H}$ & $V_{u d}$ & $V_{u s}$ & $V_{c d}$ & $V_{c s}$ & $V_{u b}$ & $V_{c b}$ \\
\hline \hline
\end{tabular}


TABLE X. Three-particle meson decay CKM matrix elements.

\begin{tabular}{ccccc}
\hline \hline$K^{0} \rightarrow \pi^{+} l^{-} N$, & $D^{0} \rightarrow K^{+} l^{-} N$, & $D^{0} \rightarrow \pi^{+} l^{-} N$, & $B^{0} \rightarrow D^{+} l^{-} N$, & $B^{0} \rightarrow \pi^{+} l^{-} N$, \\
& $D^{+} \rightarrow K^{0} l^{+} N$, & $D^{+} \rightarrow \pi^{0} l^{+} N$ & $B^{+} \rightarrow D^{0} l^{+} N$, & $B^{+} \rightarrow \pi^{0} l^{+} N$, \\
& $D^{0} \rightarrow K^{+*} l^{-} N$, & $D_{s}^{+} \rightarrow \eta^{0} l^{+} N$ & $B^{0} \rightarrow D^{+*} l^{-} N$, & $B^{+} \rightarrow \rho^{0} l^{+} N$, \\
& $D^{+} \rightarrow K^{0 *} l^{+} N$ & & $B^{+} \rightarrow D^{0 *} l^{+} N$ & $B^{0} \rightarrow \rho^{+} l^{-} N$, \\
& & & $B_{s}^{0} \rightarrow D_{s}^{+} l^{-} N$ & $B^{0} l^{-} N$, \\
& & & $B_{s}^{0} \rightarrow D_{s}^{*+} l^{-} N$ \\
\hline$V_{H H^{\prime}}$ & $V_{c s}$ & $V_{c d}$ & $V_{c b}$ \\
\hline \hline
\end{tabular}

The three-particle vector meson decays with sterile neutrino production are as follows [21]:

$$
\begin{aligned}
\frac{d \mathrm{Br}\left(H \rightarrow V l_{\alpha} N\right)}{d E_{N}}= & \tau_{H}\left|U_{\alpha}\right|^{2} C_{K}^{2} \frac{G_{F}^{2}\left|V_{H V}\right|^{2}}{32 \pi^{3} M_{H}^{2}} \int_{q_{\min }^{2}}^{q_{\max }^{2}} d q^{2}\left(\frac{f_{2}^{2}\left(q^{2}\right)}{2}\left(q^{2}-M_{N}^{2}-M_{l}^{2}+\omega^{2} \frac{\Omega^{2}-\omega^{2}}{M_{V}^{2}}\right)\right. \\
& +\frac{f_{5}^{2}\left(q^{2}\right)}{2}\left(M_{N}^{2}+M_{l}^{2}\right)\left(q^{2}-M_{N}^{2}+M_{l}^{2}\right)\left(\frac{\Omega^{4}}{4 M_{V}^{2}}-q^{2}\right)+2 f_{3}^{2}\left(q^{2}\right) M_{V}^{2}\left(\frac{\Omega^{4}}{4 M_{V}^{2}}-q^{2}\right)\left(M_{N}^{2}+M_{l}^{2}-q^{2}+\omega^{2} \frac{\Omega^{2}-\omega^{2}}{M_{V}^{2}}\right) \\
& +2 f_{3}\left(q^{2}\right) f_{5}\left(q^{2}\right)\left(M_{N}^{2} \omega^{2}+\left(\Omega^{2}-\omega^{2}\right) M_{l}^{2}\right)\left(\frac{\Omega^{4}}{4 M_{V}^{2}}-q^{2}\right)+2 f_{1}\left(q^{2}\right) f_{2}\left(q^{2}\right)\left(q^{2}\left(2 \omega^{2}-\Omega^{2}\right)+\Omega^{2}\left(M_{N}^{2}-M_{l}^{2}\right)\right) \\
& +\frac{f_{2}\left(q^{2}\right) f_{5}\left(q^{2}\right)}{2}\left(\frac{\omega^{2} \Omega^{2}}{M_{V}^{2}}\left(M_{N}^{2}-M_{l}^{2}\right)+\frac{\Omega^{4}}{M_{V}^{2}} M_{l}^{2}+2\left(M_{N}^{2}-M_{l}^{2}\right)^{2}-2 q^{2}\left(M_{N}^{2}+M_{l}^{2}\right)\right) \\
& +f_{2}\left(q^{2}\right) f_{3}\left(q^{2}\right)\left(\Omega^{2} \omega^{2} \frac{\Omega^{2}-\omega^{2}}{M_{V}^{2}}+2 \omega^{2}\left(M_{l}^{2}-M_{N}^{2}\right)+\Omega^{2}\left(M_{N}^{2}-M_{l}^{2}-q^{2}\right)\right) \\
& \left.+f_{1}^{2}\left(q^{2}\right)\left(\Omega^{4}\left(q^{2}-M_{N}^{2}+M_{l}^{2}\right)-2 M_{V}^{2}\left(q^{4}-\left(M_{N}^{2}-M_{l}^{2}\right)^{2}\right)+2 \omega^{2} \Omega^{2}\left(M_{N}^{2}-q^{2}-M_{l}^{2}\right)+2 \omega^{4} q^{2}\right)\right)
\end{aligned}
$$

where $\omega^{2}=M_{H}^{2}-M_{V}^{2}+M_{N}^{2}-M_{l}^{2}-2 M_{H} E_{N}$ and $\Omega^{2}=M_{H}^{2}-M_{V}^{2}-q^{2}, C_{K}=\frac{1}{\sqrt{2}}$ for $H^{\prime}=\rho^{0}, C_{K}=1$ for all other cases [29]; form factors $f_{i}\left(q^{2}\right)$ can be expressed via standard axial form factors $A_{0}\left(q^{2}\right), A_{1}\left(q^{2}\right), A_{2}\left(q^{2}\right)$ and vector form factor $V\left(q^{2}\right)$ as

$$
\begin{aligned}
& f_{1}\left(q^{2}\right)=\frac{V\left(q^{2}\right)}{M_{H}+M_{V}}, \quad f_{2}\left(q^{2}\right)=\left(M_{H}+M_{V}\right) A_{1}\left(q^{2}\right), \quad f_{3}\left(q^{2}\right)=-\frac{A_{2}\left(q^{2}\right)}{M_{H}+M_{V}}, \\
& f_{4}\left(q^{2}\right)=\frac{1}{q^{2}}\left(M_{V}\left(2 A_{0}-A_{1}-A_{2}\right)+M_{H}\left(A_{2}-A_{1}\right)\right), \quad f_{5}\left(q^{2}\right)=f_{3}\left(q^{2}\right)+f_{4}\left(q^{2}\right) .
\end{aligned}
$$

We take the following decays into three particles: $K_{L}^{0} \rightarrow \pi^{+} l^{-} N, K_{S}^{0} \rightarrow \pi^{+} l^{-} N, K^{+} \rightarrow \pi^{0} l^{+} N, D^{0} \rightarrow K^{+} l^{-} N$, $D^{+} \rightarrow K^{0} l^{+} N, D^{0} \rightarrow \pi^{+} l^{-} N, D^{+} \rightarrow \pi^{0} l^{+} N, D_{s}^{+} \rightarrow \eta^{0} l^{+} N, B^{+} \rightarrow D^{0} l^{+} N, B^{0} \rightarrow D^{+} l^{-} N, B^{+} \rightarrow \pi^{0} l^{+} N, B^{0} \rightarrow \pi^{+} l^{-} N$, $D^{0} \rightarrow K^{+*} l^{-} N, \quad D^{+} \rightarrow K^{0 *} l^{+} N, \quad B^{0} \rightarrow D^{+*} l^{-} N, \quad B^{+} \rightarrow D^{0 *} l^{+} N, \quad B^{+} \rightarrow \rho^{0} l^{+} N, \quad B^{0} \rightarrow \rho^{+} l^{-} N, \quad B_{s}^{0} \rightarrow D_{s}^{+} l^{-} N$, $B_{s}^{0} \rightarrow K^{+} l^{-} N, B_{s}^{0} \rightarrow D_{s}^{*+} l^{-} N, B_{s}^{0} \rightarrow K^{*+} l^{-} N$

We consider three cases: mixing with the electron neutrino $\left|U_{\mu}\right|^{2}=\left|U_{\tau}\right|^{2}=0$, mixing with the muon neutrino $\left|U_{e}\right|^{2}=\left|U_{\tau}\right|^{2}=0$, and mixing with the tau neutrino $\left|U_{e}\right|^{2}=\left|U_{\mu}\right|^{2}=0$. For the case of mixing with the tau neutrino, we have to account for the sterile neutrinos with masses $M_{N}<M_{\tau}$ produced in tauon decays. We have to consider additional processes [21]:

$$
\begin{aligned}
\frac{d \mathrm{Br}\left(\tau^{+} \rightarrow H^{+} N\right)}{d E_{N}}= & \tau_{\tau}\left|U_{\tau}\right|^{2} \frac{G_{F}^{2}\left|V_{H}\right|^{2} f_{H}^{2}}{16 \pi} M_{\tau}^{3}\left(\left(1-\frac{M_{N}^{2}}{M_{\tau}^{2}}\right)^{2}-\frac{M_{H}^{2}}{M_{\tau}^{2}}\left(1+\frac{M_{N}^{2}}{M_{\tau}^{2}}\right)\right) \sqrt{\left(1-\frac{\left(M_{H}-M_{N}\right)^{2}}{M_{\tau}^{2}}\right)\left(1-\frac{\left(M_{H}+M_{N}\right)^{2}}{M_{\tau}^{2}}\right)} \\
& \times \delta\left(E_{N}-\frac{M_{\tau}^{2}-M_{H}^{2}+M_{N}^{2}}{2 M_{\tau}}\right)
\end{aligned}
$$




$$
\begin{aligned}
\frac{d \operatorname{Br}\left(\tau^{+} \rightarrow \rho^{+} N\right)}{d E_{N}}= & \tau_{\tau}\left|U_{\tau}\right|^{2} \frac{G_{F}^{2}\left|V_{u d}\right|^{2} g_{\rho}^{2}}{8 \pi M_{\rho}^{2}} M_{\tau}^{3}\left(\left(1-\frac{M_{N}^{2}}{M_{\tau}^{2}}\right)^{2}+\frac{M_{\rho}^{2}}{M_{\tau}^{2}}\left(1+\frac{M_{N}^{2}-2 M_{\rho}^{2}}{M_{\tau}^{2}}\right)\right) \sqrt{\left(1-\frac{\left(M_{\rho}-M_{N}\right)^{2}}{M_{\tau}^{2}}\right)\left(1-\frac{\left(M_{\rho}+M_{N}\right)^{2}}{M_{\tau}^{2}}\right)} \\
\times & \delta\left(E_{N}-\frac{M_{\tau}^{2}-M_{\rho}^{2}+M_{N}^{2}}{2 M_{\tau}}\right), \\
\frac{d \operatorname{Br}\left(\tau^{+} \rightarrow \nu_{\alpha} l_{\alpha}^{+} N\right)}{d E_{N}}= & \tau_{\tau}\left|U_{\tau}\right|^{2} \frac{G_{F}^{2}}{4 \pi^{3}} M_{\tau}^{2}\left(1-\frac{M_{l}^{2}}{M_{\tau}^{2}+M_{N}^{2}-2 E_{N} M_{\tau}}\right)^{2} \sqrt{E_{N}^{2}-M_{N}^{2}}\left(\left(M_{\tau}-E_{N}\right)\left(1-\frac{M_{N}^{2}+M_{l}^{2}}{M_{\tau}^{2}}\right)\right. \\
& \left.-\left(1-\frac{M_{l}^{2}}{M_{\tau}^{2}+M_{N}^{2}-2 E_{N} M_{\tau}}\right)\left(\frac{\left(M_{\tau}-E_{N}\right)^{2}}{M_{\tau}}+\frac{E_{N}^{2}-M_{N}^{2}}{3 M_{\tau}}\right)\right) ;
\end{aligned}
$$

here $H \in\left\{\pi^{+}, K^{+}\right\}, \alpha \in\{e, \mu\}$. Note that if the sterile neutrino mixes not only with tau neutrino but also with the electron neutrino or muon neutrino, then another process, $\tau \rightarrow \nu_{\tau} l_{\alpha} N$, becomes available.

To account for the processes (B17)-(B19), one has to study the processes in which tauons are produced. Tauons are produced in the decays of heavy mesons, with the most significant ones being $D_{s}^{+} \rightarrow \tau^{+} \nu_{\tau}, \quad B^{0} \rightarrow D^{-} \tau^{+} \nu_{\tau}$, $B^{0} \rightarrow D^{*-} \tau^{+} \nu_{\tau}, B^{+} \rightarrow \bar{D}^{0} \tau^{+} \nu_{\tau}, B^{+} \rightarrow \bar{D}^{* 0} \tau^{+} \nu_{\tau}$ [27]. The branching ratios for the tauon production coincide with the ones described by Eqs. (B8), (B9), (B15), and (B17)(B19), where sterile neutrino $N$ and lepton $l$ are replaced by tauon $\tau$ and active neutrino $\nu_{\tau}$. One can obtain the resulting tauon momentum and coordinates with formulas (12)-(16) in the same way.

To obtain $\operatorname{Br}(H \rightarrow N \ldots)$, one needs to integrate Eqs. (B8), (B9), (B15), and (B17)-(B19) over $E_{N}$ from $M_{N}$ to $\frac{1}{2 M_{H}}\left(M_{H}^{2}+M_{N}^{2}-\left(M_{H^{\prime}}+M_{l}\right)^{2}\right)$. Note that other processes that may contribute to neutrino production have been studied [29]. The most important of them are multimeson decay channels. For a heavier sterile neutrino, their importance becomes more significant. By ignoring these states, we can underestimate the total inclusive width $\frac{1}{\tau_{H}} \times \sum_{X} \operatorname{Br}(H \rightarrow N X)$ by about 20\% [29]. On the other hand, as seen in Figs. 2 and 3, at $M_{N} \gtrsim 2 \mathrm{GeV}$, too few sterile neutrinos reach the Near Detector to be successfully registered. For $M_{N}<2 \mathrm{GeV}$, the multimeson channels do not contribute significantly.

We note that many of the mentioned processes also prove to be insignificant in the interesting mass range. To determine this, we calculate the contribution of each process to sterile neutrino production. If the process's branching value is less than $1 \%$ for all considered values of sterile neutrino mass, then it is taken out of consideration. This way, the following processes were considered insignificant: $K_{S}^{0} \rightarrow \pi^{+} l^{-} N, B^{-} \rightarrow \pi^{0} l^{-} N, B^{0} \rightarrow \pi^{+} l^{-} N$, $B_{s}^{0} \rightarrow K^{+} l^{-} N, B^{0} \rightarrow \rho^{+} l^{-} N, B^{-} \rightarrow \rho^{0} l^{-} N, B_{s}^{0} \rightarrow K^{*+} l^{-} N$. We note that, for the case of a mixing with a tau neutrino, only $D_{s}$ and heavier mesons can kinematically simultaneously produce the sterile neutrino and tauon in their decays (if $M_{H}>M_{\tau}+M_{N}$ ).
[1] P. Minkowski, $\mu \rightarrow e \gamma$ at a rate of one out of $10^{9}$ muon decays?, Phys. Lett. 67B, 421 (1977); see also, M. GellMann, P. Ramond, and R. Slansky, Complex spinors and unified theories, Conf. Proc. C 790927, 315 (1979); R. N. Mohapatra and G. Senjanovic, Neutrino Mass and Spontaneous Parity Violation, Phys. Rev. Lett. 44, 912 (1980).

[2] A. Boyarsky, O. Ruchayskiy, and M. Shaposhnikov, The role of sterile neutrinos in cosmology and astrophysics, Annu. Rev. Nucl. Part. Sci. 59, 191 (2009).

[3] M. Drewes et al., A white paper on keV sterile neutrino dark matter, J. Cosmol. Astropart. Phys. 01 (2017) 025.

[4] F. Bergsma et al. (CHARM Collaboration), A search for decays of heavy neutrinos in the mass range $0.5-2.8 \mathrm{GeV}$, Phys. Lett. 166B, 473 (1986).
[5] A. Vaitaitis et al. (NuTeV and E815 Collaborations), Search for Neutral Heavy Leptons in a High-Energy Neutrino Beam, Phys. Rev. Lett. 83, 4943 (1999).

[6] G. Bernardi et al., Further limits on heavy neutrino couplings, Phys. Lett. B 203, 332 (1988).

[7] P. Abreu et al. (DELPHI Collaboration), Search for neutral heavy leptons produced in $Z$ decays, Z. Phys. C 74, 57 (1997); Erratum, Z. Phys. C 75, 580(E) (1997).

[8] A. S. Sadovsky et al. (OKA Collaboration), Search for heavy neutrino in $K^{+} \rightarrow \mu^{+} \nu_{H}$ decay, Eur. Phys. J. C 78, 92 (2018).

[9] S. Antusch, E. Cazzato, and O. Fischer, Sterile neutrino searches via displaced vertices at LHCb, Phys. Lett. B 774, 114 (2017). 
[10] L. Canetti, M. Drewes, and B. Garbrecht, Probing leptogenesis with $\mathrm{GeV}$-scale sterile neutrinos at $\mathrm{LHCb}$ and Belle II, Phys. Rev. D 90, 125005 (2014).

[11] A. V. Artamonov et al. (E949 Collaboration), Search for heavy neutrinos in $K^{+} \rightarrow \mu^{+} \nu_{H}$ decays, Phys. Rev. D 91, 052001 (2015); Erratum, Phys. Rev. D 91, 059903(E) (2015).

[12] E. Cortina Gil et al. (NA62 Collaboration), Search for heavy neutral lepton production in $K^{+}$decays, Phys. Lett. B 778, 137 (2018).

[13] M. Drewes, J. Hajer, J. Klaric, and G. Lanfranchi, Perspectives to find heavy neutrinos with NA62, arXiv:1806.00100.

[14] C. Ahdida et al. (SHiP Collaboration), Sensitivity of the SHiP experiment to heavy neutral leptons, J. High Energy Phys. 04 (2019) 077.

[15] D. Curtin et al., Long-lived particles at the energy frontier: the MATHUSLA physics case, Rep. Prog. Phys. 82, 116201 (2019).

[16] A. Izmaylov and S. Suvorov, Search for heavy neutrinos in the ND280 near detector of the T2K experiment, Phys. Part. Nucl. 48, 984 (2017).

[17] R. Acciarri et al. (DUNE Collaboration), Long-Baseline Neutrino Facility (LBNF) and Deep Underground Neutrino Experiment (DUNE) : Conceptual Design Report, Volume 1: The LBNF and DUNE Projects, arXiv:1601 .05471 .

[18] R. Acciarri et al. (DUNE Collaboration), Long-Baseline Neutrino Facility (LBNF) and Deep Underground Neutrino Experiment (DUNE) : Conceptual Design Report, Volume 2: The Physics Program for DUNE at LBNF, arXiv:1512 .06148 .

[19] J. Strait et al. (DUNE Collaboration), Long-Baseline Neutrino Facility (LBNF) and Deep Underground Neutrino Experiment (DUNE) : Conceptual Design Report, Volume 3: Long-Baseline Neutrino Facility for DUNE, arXiv:1601 .05823 .
[20] R. Acciarri et al. (DUNE Collaboration), Long-Baseline Neutrino Facility (LBNF) and Deep Underground] Neutrino Experiment (DUNE) : Conceptual Design Report, Volume 4 The DUNE Detectors at LBNF, arXiv:1601.02984.

[21] D. Gorbunov and M. Shaposhnikov, How to find neutral leptons of the $\nu$ MSM?, J. High Energy Phys. 10 (2007) 015; Erratum, J. High Energy Phys. 11 (2013) 101(E).

[22] S. N. Gninenko, D. S. Gorbunov, and M. E. Shaposhnikov, Search for $\mathrm{GeV}$-scale sterile neutrinos responsible for active neutrino oscillations and baryon asymmetry of the Universe, Adv. High Energy Phys. (London) 2012, 718259 (2012).

[23] C. Adams et al. (LBNE Collaboration), The long-baseline neutrino experiment: exploring fundamental symmetries of the universe, arXiv:1307.7335.

[24] K. Kodama et al. (Fermilab E653 Collaboration), Charm meson production in $800-\mathrm{GeV} / \mathrm{c}$ proton - emulsion interactions, Phys. Lett. B 263, 573 (1991).

[25] M. Aguilar-Benitez et al. (LEBC-EHS Collaboration), D Meson production from $400 \mathrm{GeV} / \mathrm{cpp}$ interactions, Phys. Lett. B 189, 476 (1987); Erratum, Phys. Lett. B 208, 530(E) (1988).

[26] See http://home.thep.lu.se/ torbjorn/pythia82html/ Fragmentation.html.

[27] M. Tanabashi et al. (Particle Data Group), Review of particle physics, Phys. Rev. D 98, 030001 (2018).

[28] C. Lourenco and H. K. Wohri, Heavy flavour hadroproduction from fixed-target to collider energies, Phys. Rep. 433, 127 (2006).

[29] K. Bondarenko, A. Boyarsky, D. Gorbunov, and O. Ruchayskiy, Phenomenology of GeV-scale heavy neutral leptons, J. High Energy Phys. 11 (2018) 032.

[30] S. Alekhin et al., A facility to search for hidden particles at the CERN SPS: the SHiP physics case, Rep. Prog. Phys. 79, 124201 (2016).

[31] A. Ariga et al. (FASER Collaboration), FASER's physics reach for long-lived particles, Phys. Rev. D99, 095011 (2019). 